\title{
DIFFERENTIAL FORMS ON REGULAR AFFINE ALGEBRAS
}

\author{
BY

\section{G. HOCHSCHILD, BERTRAM KOSTANT AND ALEX ROSENBERG( $\left.{ }^{1}\right)$}

1. Introduction. The formal apparatus of the algebra of differential forms appears as a rather special amalgam of multilinear and homological algebra, which has not been satisfactorily absorbed in the general theory of derived functors. It is our main purpose here to identify the exterior algebra of differential forms as a certain canonical graded algebra based on the Tor functor and to obtain the cohomology of differential forms from the Ext functor of a universal algebra of differential operators similar to the universal enveloping algebra of a Lie algebra.

Let $K$ be a field, $R$ a commutative $K$-algebra, $T_{R}$ the $R$-module of all $K$-derivations of $R, D_{R}$ the $R$-module of the formal $K$-differentials (see $\S 4$ ) on $R$. It is an immediate consequence of the definitions that $T_{R}$ may be identified with $\operatorname{Hom}_{R}\left(D_{R}, R\right)$. However, in general, $D_{R}$ is not identifiable with $\operatorname{Hom}_{R}\left(T_{R}, R\right)$. The algebra of the formal differentials is the exterior $R$ algebra $E\left(D_{R}\right)$ built over the $R$-module $D_{R}$. The algebra of the differential forms is the $R$-algebra $\operatorname{Hom}_{R}\left(E\left(T_{R}\right), R\right)$, where $E\left(T_{R}\right)$ is the exterior $R$-algebra built over $T_{R}$ and where the product is the usual "shuffle" product of alternating multilinear maps.

The point of departure of our investigation lies in the well-known and elementary observation that $T_{R}$ and $D_{R}$ are naturally isomorphic with $\operatorname{Ext}_{R^{e}}^{1}(R, R)$ and $\operatorname{Tor}_{1}^{R^{e}}(R, R)$, respectively, where $R^{e}=R \otimes_{K} R$. Moreover, both $\operatorname{Ext}_{R^{e}}(R, R)$ and $\operatorname{Tor}^{R^{e}}(R, R)$ can be equipped in a natural fashion with the structure of a graded skew-commutative $R$-algebra, and there is a natural duality homomorphism $h: \operatorname{Ext}_{R^{e}}(R, R) \rightarrow \operatorname{Hom}_{R}\left(\operatorname{Tor}^{R^{e}}(R, R), R\right)$, which extends the natural isomorphism of $T_{R}$ onto $\operatorname{Hom}_{R}\left(D_{R}, R\right)$.

We concentrate our attention chiefly on a regular affine $K$-algebra $R$ (cf. $\S 2$ ), where $K$ is a perfect field. Our first main result is that then the algebra $\operatorname{Tor}^{R^{e}}(R, R)$ coincides with the algebra $E\left(D_{R}\right)$ of the formal differentials, $\operatorname{Ext}_{R^{\bullet}}(R, R)$ coincides with $E\left(T_{R}\right)$, and the above duality homomorphism $h$ is an isomorphism dualizing into an isomorphism of the algebra $E\left(D_{R}\right)$ of the formal differentials onto the algebra $\operatorname{Hom}_{R}\left(E\left(T_{R}\right), R\right)$ of the differential forms.

In order to identify the cohomology of differential forms with an Ext functor, we construct a universal "algebra of differential operators," $V_{R}$,

Received by the editors May $5,1961$.

(1) Written while B. Kostant was partially supported by Contract AF49(638)-79 and A. Rosenberg by N.S.F. Grant G-9508. 
which is the universal associative algebra for the representations of the $K$-Lie algebra $T_{R}$ on $R$-modules in which the $R$-module structure and the $T_{R}$-module structure are tied together in the natural fashion. After establishing a number of results on the structure and representation theory of $V_{R}$, we show that, under suitable assumptions on the $K$-algebra $R$ and, in particular, if $R$ is a regular affine $K$-algebra where $K$ is a perfect field, the cohomology $K$-algebra derived from the differential forms may be identified with $\operatorname{Ext}_{V_{R}}(R, R)$.

In $\S 2$, we show that the tensor product of two regular affine algebras over a perfect field is a regular ring, and we prove a similar result for tensor products of fields. $\S \S 3,4$ and 5 include, besides the proof of the first main result, a study of the formal properties of the Tor and Ext algebras and the pairing between them, for general commutative algebras. In the remainder of this paper, we deal with the universal algebra $V_{R}$ of differential operators. In particular, we prove an analogue of the Poincaré-Birkhoff-Witt Theorem, which is needed for obtaining an explicit projective resolution of $R$ as a $V_{R}$-module. Also, we discuss the homological dimensions connected with $V_{R}$.

We have had advice from $M$. Rosenlicht on several points of an algebraic geometric nature, and we take this opportunity to express our thanks to him.

2. Regular rings. Let $R$ be a commutative ring and let $P$ be a prime ideal of $R$. We denote the corresponding ring of quotients by $R_{P}$. The elements of $R_{P}$ are the equivalence classes of the pairs $(x, y)$, where $x$ and $y$ are elements of $R$, and $y$ does not lie in $P$, and where two pairs $\left(x_{1}, y_{1}\right)$ and $\left(x_{2}, y_{2}\right)$ are called equivalent if there is an element $z$ in $R$ such that $z$ does not lie in $P$ and $z\left(x_{1} y_{2}-x_{2} y_{1}\right)=0$.

By the Krull dimension of $R$ is meant the largest non-negative integer $k$ (or $\infty$, if there is no largest one) for which there is a chain of prime ideals, with proper inclusions, $P_{0} \subset \cdots \subset P_{k} \subset R$. A Noetherian local ring always has finite Krull dimension, and it is called a regular local ring if its maximal ideal can be generated by $k$ elements, where $k$ is the Krull dimension. A commutative Noetherian ring $R$ with identity element is said to be regular if, for every maximal ideal $P$ of $R$, the corresponding ring of quotients $R_{P}$ is a regular local ring $[2, \S 4]$.

It is well known that a regular local ring is an integrally closed integral domain [14, Cor. 1, p. 302]. It follows that a regular integral domain $R$ is integrally closed; for, if $x$ is an element of the field of quotients of $R$ that is integral over $R$ then $x \in R_{P}$, for every maximal ideal $P$ of $R$, which evidently implies that $x \in R$.

Let $K$ be a field. By an affine $K$-algebra is meant an integral domain $R$ containing $K$ and finitely ring-generated over $K$. An affine $K$-algebra is Noetherian, and its Krull dimension is equal to the transcendence degree of its field of quotients over $K$, and the same holds for the Krull dimension of every one of its rings of quotients with respect to maximal ideals $[14, \mathrm{Ch}$. VII, §7]. 
THEOREM 2.1( $\left.{ }^{2}\right)$. Let $K$ be a perfect field, and let $R$ and $S$ be regular affine $K$-algebras. Then $R \otimes_{K} S$ is regular.

Proof. Suppose first that $R \otimes_{K} S$ is an integral domain, and let $M$ be one of its maximal ideals. Put $M_{1}=(M \cap R) \otimes_{K} S+R \otimes_{K}(M \cap S)$. Then $M_{1}$ is an ideal of $R \otimes_{K} S$ that is contained in $M$, and we have $\left(R \otimes_{K} S\right) / M_{1}$ $=(R /(M \cap R)) \otimes_{K}(S /(M \cap S))$. Now $R /(R \cap M)$ and $S /(M \cap S)$ are subrings of $\left(R \otimes_{K} S\right) / M$ containing $K$. Since $\left(R \otimes_{K} S\right) / M$ is a finite algebraic extension field of $K$, the same is therefore true for $R /(M \cap R)$ and $S /(M \cap S)$. Since $K$ is perfect, it follows that we have a direct $K$-algebra decomposition $\left(R \otimes_{K} S\right) / M_{1}=U+M / M_{1}$. Let $z$ be a representative in $R \otimes_{K} S$ of a nonzero element of $U$. Then $z$ does not belong to $M$, and $z M \subset M_{1}$. Hence it is clear that $M\left(R \otimes_{K} S\right)_{M}=M_{1}\left(R \otimes_{K} S\right)_{M}$.

Since $R$ is regular, the maximal ideal $(M \cap R) R_{M \cap R}$ of the local ring $R_{M \cap R}$ is generated by $d_{R}$ elements, where $d_{R}$ is the degree of transcendence of the field of quotients of $R$ over $K$. Similarly, $(M \cap S) S_{M \cap S}$ is generated by $d_{S}$ elements, where $d_{S}$ is the degree of transcendence of the quotient field of $S$ over $K$. These $d_{R}+d_{S}$ elements may be regarded as elements of $\left(R \otimes_{K} S\right)_{M}$ and evidently generate the ideal $M_{1}\left(R \otimes_{K} S_{J_{M}}\right.$. Hence we conclude that the maximal ideal of $\left(R \otimes_{K} S\right)_{M}$ can be generated by $d_{R}+d_{S}$ elements. Since the degree of transcendence of the quotient field of $R \otimes_{K} S$ over $K$ is equal to $d_{R}+d_{S}$, this means that $\left(R \otimes_{K} S\right)_{M}$ is a regular local ring. Thus $R \otimes_{K} S$ is regular.

Now let us consider the general case. Let $Q(R)$ and $Q(S)$ denote the fields of quotients of $R$ and $S$. Let $K^{R}$ and $K^{S}$ be the algebraic closures of $K$ in $Q(R)$ and in $Q(S)$, respectively. Since $R$ and $S$ are integrally closed, we have $K^{R} \subset R$ and $K^{S} \subset S$. Since $Q(R)$ and $Q(S)$ are finitely generated extension fields of $K$, so are $K^{R}$ and $K^{S}$. Thus $K^{R}$ and $K^{S}$ are finite algebraic extensions of $K$.

Let $M$ be a maximal ideal of $R \otimes_{K} S$. Since $K$ is perfect, we have a direct $K$-algebra decomposition $K^{R} \otimes_{K} K^{S}=U+M_{1}$, where $M_{1}=M \cap\left(K^{R} \otimes_{K} K^{S}\right)$. Hence we have

$$
R \otimes_{K} S=R \otimes_{K^{R}}\left(K^{R} \otimes_{K} K^{S}\right) \otimes_{K^{S}} S=R \otimes_{K^{R}} U \otimes_{K^{S}} S+M_{2},
$$

where the last sum is a direct $K$-algebra sum, and $M_{2}=R \otimes_{K^{R}} M_{1} \otimes_{K^{s}} S \subset M$.

Evidently, $U$ may be identified with a subring of the field $\left(R \otimes_{K} S\right) / M$ containing $K$. Hence $U$ is a finite algebraic extension field of $K$. Identifying $K^{R}$ and $K^{S}$ with their images in $U$, we may also regard $U$ as a finite algebraic extension field of $K^{R}$ or $K^{S}$. Since $K$ is perfect, $U$ is generated by a single element over $K^{R}$ or over $K^{S}$. The minimum polynomial of this element over $K^{R}$ or over $K^{S}$ remains irreducible in $Q(R)[x]$ or in $Q(S)[x]$, because $K^{R}$ is algebraically closed in $Q(R)$ and $K^{S}$ is algebraically closed in $Q(S)$. Hence

(2) The referee informs us that this result is an immediate consequence of cohomology results obtained by D. K. Harrison in a paper on Commutative algebras and cohomology, to appear in these Transactions. 
$R \otimes_{K^{R}} U$ and $U \otimes_{K^{s}} S$ are integral domains. Moreover, by the part of the theorem we have already proved, they are regular.

Let $T$ denote the field $U \otimes_{K^{s}} Q(S)$. This is a finitely generated extension field of the perfect field $K^{R}$. Let $\left(t_{1}, \cdots, t_{n}\right)$ be a separating transcendence base for $T$ over $K^{R}$, and put $T_{0}=K^{R}\left(t_{1}, \cdots, t_{n}\right)$. We have $Q(R) \otimes_{K^{R}} T$ $=\left(Q(R) \otimes_{K^{R}} T_{0}\right) \otimes_{T_{0}} T$, and we may identify $Q(R) \otimes_{K^{R}} T_{0}$ with a subring of $Q(R)\left(t_{1}, \cdots, t_{n}\right)$, with $\left(t_{1}, \cdots, t_{n}\right)$ algebraically free over $Q(R)$. Since $K^{R}$ is algebraically closed in $Q(R)$, it follows that $K^{R}\left(t_{1}, \cdots, t_{n}\right)$ is algebraically closed in $Q(R)\left(t_{1}, \cdots, t_{n}\right)[6$, Lemma 2, p. 83]. Now it follows by the argument we made above that $R \otimes_{K^{R}} T$ is an integral domain, so that $R \otimes_{K^{R}} U \otimes_{K^{s}} S$ is an integral domain. On the other hand, this is the tensor product, relative to the perfect field $U$, of the regular affine $U$-algebras $R \otimes_{K^{R}} U$ and $U \otimes_{K^{s}} S$. Hence we may conclude from what we have already proved that $R \otimes_{K^{R}} U \otimes_{K^{s}} S$ is regular.

Now consider the direct $K$-algebra decomposition

$$
R \otimes_{K} S=R \otimes_{K^{R}} U \otimes_{K^{s}} S+M_{2} .
$$

Since $M_{2} \subset M$, the corresponding projection epimorphism $R \otimes_{K} S$ $\rightarrow R \otimes_{K^{R}} U \otimes_{K^{s}} S$ sends the complement of $M$ in $R \otimes_{K} S$ onto the complement of $M \cap\left(R \otimes_{K^{R}} U \otimes_{K^{s}} S\right)$ in $R \otimes_{K^{R}} U \otimes_{K^{s}} S$. Moreover, there is an element $z$ in the complement of $M$ such that $z M_{2}=(0)$. Hence it is clear that the projection epimorphism yields an isomorphism of $\left(R \otimes_{K} S\right)_{M}$ onto the local ring over $R \otimes_{K^{R}} U \otimes_{K^{S}} S$ that corresponds to the maximal ideal $M \cap\left(R \otimes_{K^{R}} U \otimes_{K} s S\right)$. Hence $\left(R \otimes_{K} S\right)_{M}$ is a regular local ring, and Theorem 2.1 is proved.

THEOREM 2.2. Let $K$ be an arbitrary field, let $F$ be a finitely and separably generated extension field of $K$, and let $L$ be an arbitrary field containing $K$. Then $F \otimes_{K} L$ is a regular ring.

Proof. It is known that the (homological) algebra $\operatorname{dimension} \operatorname{dim}(F)$, i.e., the projective dimension of $F$ as an $F \otimes_{K} F$-module is finite; in fact, it is equal to the transcendence degree of $F$ over $K\left[11\right.$, Th. 10]. Since $\operatorname{dim}\left(F \otimes_{K} L\right)$ $=\operatorname{dim}(F)$, where $F \otimes_{K} L$ is regarded as an $L$-algebra [4, Cor. 7.2, p. 177] we have that $\operatorname{dim}\left(F \otimes_{K} L\right)$ is finite. Since $L$ is a field, this implies that the global homological dimension $d\left(F \otimes_{K} L\right)$ is also finite [4, Prop. 7.6, p. 179]. Since $F \otimes_{K} L$ is a commutative Noetherian ring, we have, for every maximal ideal $M$ of $F \otimes_{K} L, d\left(\left(F \otimes_{K} L\right)_{M}\right) \leqq d\left(F \otimes_{K} L\right)$ [4, Ex. 11, p. 142; 1, Th. 1]. Thus each local ring $\left(F \otimes_{K} L\right)_{M}$ is of finite global homological dimension. By a well-known result of Serre's [12, Th. 3], this implies that $\left(F \otimes_{K} L\right)_{M}$ is a regular local ring. Hence $F \otimes_{K} L$ is a regular ring.

Note. Actually, we shall later appeal only to the following special consequence of Theorem 2.2: let $F$ be a finitely separably generated extension field of $K$; let $J$ be the kernel of the natural epimorphism $F \otimes_{K} F \rightarrow F$; then the 
local ring $\left(F \otimes_{K} F\right)_{J}$ is regular. This special result can be proved much more easily and directly along the lines of our proof of Theorem 2.1. On the other hand, Theorem 2.1 can be derived more quickly, though less elementarily, from the result of Serre used above.

3. The Tor-algebra for regular rings. Let $R$ and $S$ be commutative rings with identity elements, and let $\phi$ be a unitary ring epimorphism $S \rightarrow R$. We regard $R$ as a right or left $S$-module via $\phi$, in the usual way, and we consider $\operatorname{Tor}^{S}(R, R)$.

Since $S$ is commutative, every left $S$-module may also be regarded as a right $S$-module, and we shall do so whenever this is convenient. Let $H$ stand for the homology functor on complexes of $S$-modules, and let $U$ and $V$ be any two $S$-module complexes. There is an evident canonical homomorphism of $H(U) \otimes_{S} H(V)$ into $H\left(U \otimes_{S} V\right)$, which gives rise to an algebra structure on $\operatorname{Tor}^{S}(R, R)$, as follows. Let $X$ be an $S$-projective resolution of $R$. With $U=V=R \otimes_{S} X$, the canonical homomorphism becomes a homomorphism

$$
\operatorname{Tor}^{S}(R, R) \otimes_{S} \operatorname{Tor}^{S}(R, R) \rightarrow H\left(\left(R \otimes_{S} X\right) \otimes_{S}\left(R \otimes_{S} X\right)\right) .
$$

Evidently, $\left(R \otimes_{S} X\right) \otimes_{S}\left(R \otimes_{S} X\right)$ may be identified with $\left(R \otimes_{S} R\right) \otimes_{S}\left(X \otimes_{S} X\right)$, and hence with $R \otimes_{S}\left(X \otimes_{S} X\right)$. Now $X \otimes_{S} X$ is an $S$-projective complex over $R \otimes_{S} R=R$, whence we have the natural homomorphism

$$
H\left(R \otimes_{S}\left(X \otimes_{S} X\right)\right) \rightarrow \operatorname{Tor}^{S}(R, R) .
$$

Composing this with the homomorphism above, we obtain an $S$-module homomorphism

$$
\operatorname{Tor}^{S}(R, R) \otimes_{S} \operatorname{Tor}^{S}(R, R) \rightarrow \operatorname{Tor}^{S}(R, R) .
$$

This is the product $\cap$ of $[4$, p. 211] and it is independent of the choice of the resolution $X$. Standard arguments on tensor products of complexes and resolutions show that this product is associative and skew-commutative in the sense that $\alpha \beta=(-1)^{p q} \beta \alpha$ when $\alpha$ is homogeneous of degree $p$ and $\beta$ is homogeneous of degree $q$. In principle, this product is a product of $S$-algebras. However, $S$ operates on $\operatorname{Tor}^{S}(R, R)$ through $\phi: S \rightarrow R$, and we shall accordingly regard $\operatorname{Tor}^{S}(R, R)$ as an $R$-algebra.

THEOREM 3.1. Let $S$ and $R$ be Noetherian commutative rings with identity elements, and let $\phi$ be a ring epimorphism $S \rightarrow R$ with kernel $I$. Assume that $R$ is a regular ring and that, for every maximal ideal $M$ of $S$ that contains $I$, the local ring $S_{M}$ is regular. Then $\operatorname{Tor}^{S}(R, R)$ is finitely generated and projective as an $R$-module and is naturally isomorphic with the exterior $R$-algebra constructed over $\operatorname{Tor}_{1}^{S}(R, R)$.

Proof. Let $T$ denote the tensor algebra constructed over the $R$-module $\operatorname{Tor}_{1}^{S}(R, R)$, let $P$ denote the kernel of the canonical $R$-algebra homomorphism 
$\psi: T \rightarrow \operatorname{Tor}^{S}(R, R)$, and put $Q=\operatorname{Tor}^{S}(R, R) / \psi(T)$. Let $U$ denote the 2 -sided ideal of $T$ that is generated by the squares of the elements of $\operatorname{Tor}_{1}^{S}(R, R)$. The last assertion of our theorem means that $Q=(0)$ and $P=U$. The statement $Q=(0)$ is equivalent to the statement $R_{N} \otimes_{R} Q=(0)$, for all maximal ideals $N$ of $R$. The statement $P=U$ is equivalent to the statement $(P+U) / P=(0)$ and $(P+U) / U=(0)$, or to the statement $R_{N} \otimes_{R}(P+U) / P=(0)$ and $R_{N} \otimes_{R}(P+U) / U=(0)$, for all maximal ideals $N$ of $R$. This, in turn, is equivalent to the statement that the images of $R_{N} \otimes_{R} P$ and $R_{N} \otimes_{R} U$ in $R_{N} \otimes_{R}(P+U)$ coincide with $R_{N} \otimes_{R}(P+U)$. Since $R_{N}$ is $R$-flat, these tensor products may be identified with their canonical images in $R_{N} \otimes_{R} T$; and $R_{N} \otimes_{R} P$ is thereby identified with the kernel of the homomorphism of $R_{N} \otimes_{R} T$ into $R_{N} \otimes_{R} \operatorname{Tor}^{S}(R, R)$ that is induced by $\psi$. Hence it is clear that the statement $Q=(0)$ and $P=U$ is equivalent to the statement that the homomorphism of $R_{N} \otimes_{R} T$ into $R_{N} \otimes_{R} \operatorname{Tor}^{S}(R, R)$ that is induced by $\psi$ is an epimorphism with kernel $R_{N} \otimes_{R} U$, for every maximal ideal $N$ of $R$.

Let $M$ be the maximal ideal of $S$ that contains $I$ and is such that $M / I=N$. Clearly, the epimorphism $\phi$ induces an epimorphism $S_{M} \rightarrow R_{N}$ with kernel $I S_{M}$ in the natural fashion.

Now let $X$ be an $S$-projective resolution of $R$. Since $S_{M}$ is $S$-flat, $S_{M} \otimes_{S} X$ is then an $S_{M}$-projective resolution of $S_{M} \otimes_{S} R=S_{M} / I S_{M}=R_{N}$. Hence we have

$$
\operatorname{Tor}^{S_{M}}\left(S_{M} / I S_{M}, S_{M} / I S_{M}\right)=H\left(\left(S_{M} / I S_{M}\right) \otimes_{S_{M}}\left(S_{M} \otimes_{S} X\right)\right) .
$$

On the other hand,

$\left(S_{M} / I S_{M}\right) \otimes_{S_{M}}\left(S_{M} \otimes_{S} X\right)=\left(S_{M} / I S_{M}\right) \otimes_{S} X=R_{N} \otimes_{S} X=R_{N} \otimes_{R}\left(R \otimes_{S} X\right)$.

Since $R_{N}$ is $R$-flat, we have $H\left(R_{N} \otimes_{R}\left(R \otimes_{S} X\right)\right)=R_{N} \otimes_{R} \operatorname{Tor}^{S}(R, R)$. Thus $R_{N} \otimes_{R} \operatorname{Tor}^{S}(R, R)$ is naturally isomorphic with $\operatorname{Tor}^{S_{M}}\left(S_{M} / I S_{M}, S_{M} / I S_{M}\right)$. Similarly, we see that $R_{N} \otimes_{R} T$ is naturally isomorphic with the tensor algebra constructed over the $R_{N}$-module $\operatorname{Tor}_{1}^{S_{M}}\left(S_{M} / I S_{M}, S_{M} / I S_{M}\right)$. Moreover, it is easily seen that these isomorphisms transport our homomorphism $R_{N} \otimes_{R} T$ $\rightarrow R_{N} \otimes_{R} \operatorname{Tor}^{S}(R, R)$ into the canonical homomorphism of the tensor algebra over $\operatorname{Tor}_{1}^{S_{M}}\left(S_{M} / I S_{M}, S_{M} / I S_{M}\right)$ into $\operatorname{Tor}^{S_{M}}\left(S_{M} / I S_{M}, S_{M} / I S_{M}\right)$.

Each $\operatorname{Tor}_{p}^{S}(R, R)$ is finitely generated as an $S$-module, and hence also as an $R$-module. Hence if we show that $R_{N} \otimes_{R} \operatorname{Tor}_{p}^{\mathcal{S}}(R, R)$ is a free $R_{N}$-module, for every maximal ideal $N$ of $R$, we shall be able to conclude from a standard result [4, Ex. 11, p. 142] that $\operatorname{Tor}_{p}^{S}(R, R)$ is a finitely generated projective $R$-module. In particular, if $\operatorname{Tor}_{1}^{\mathcal{S}}(R, R)$ is a finitely generated projective $R$ module, we imbed it as a direct $R$-module summand in a finitely generated free $R$-module to show that the exterior algebra constructed over it has nonzero components only up to a certain degree and is a finitely generated projective $R$-module.

From this preparation, it is clear that it suffices to adduce the following 
result $\left({ }^{3}\right)$ : let $L\left(=S_{M}\right)$ be a regular local ring and let $J\left(=I S_{M}\right)$ be a prime ideal of $L$ such that the local ring $L / J$ is regular. Then $\operatorname{Tor}_{1}^{L}(L / J, L / J)$ is a finitely generated free $L / J$-module, and $\operatorname{Tor}^{L}(L / J, L / J)$ is naturally isomorphic, as an $L / J$-algebra, with the exterior algebra constructed over $\operatorname{Tor}_{1}^{L}(L / J, L / J)$.

To prove this, note first that the assumptions imply that the ideal $J$ can be generated by an $L$-sequence $\left(a_{1}, \cdots, a_{j}\right)$ of elements of $L$, i.e., by a system with the property that each $a_{k}$ is not a zero-divisor mod the ideal generated by $a_{1}, \cdots, a_{k-1}[14$, Th. 26, p. 303 and Cor. 1, p. 302]. If $X$ is the Koszul resolution of $L / J$ as an $L$-module [4, pp. 151-153], constructed with the use of this $L$-sequence, then $X$ has the structure of an exterior $L$-algebra over a free $L$-module of rank $j$, this algebra structure being compatible with the boundary map, so that it induces the algebra structure on $\operatorname{Tor}^{L}(L / J, L / J)$ via $(L / J) \otimes_{L} X$. Moreover, the boundary map on $(L / J) \otimes_{L} X$ is the zero map. Hence it follows immediately that $\operatorname{Tor}_{1}^{L}(L / J, L / J)$ is a free $L / J$-module of rank $j$ and that $\operatorname{Tor}^{L}(L / J, L / J)$ is the exterior algebra over this module. This completes the proof of Theorem 3.1.

4. Duality between Tor and Ext. Let $R$ and $S$ be commutative rings with identity elements, and let $\phi$ be a ring epimorphism of $S$ onto $R$. As before, all $R$-modules are regarded as $S$-modules via $\phi$. Let $X$ be an $S$-projective resolution of $R$, and let $A$ be an $R$-module. Then $\operatorname{Ext}_{S}(R, A)=H\left(\operatorname{Hom}_{S}(X, A)\right)$. Clearly, we may identify $\operatorname{Hom}_{S}(X, A)$ with $\operatorname{Hom}_{R}\left(R \otimes_{S} X, A\right)$, so that we may write $\operatorname{Ext}_{S}(R, A)=H\left(\operatorname{Hom}_{R}\left(R \otimes_{S} X, A\right)\right)$. Now there is a natural map (a specialization of $[4$, p. 119 , last line $]$ )

$$
h: H\left(\operatorname{Hom}_{R}\left(R \otimes_{S} X, A\right)\right) \rightarrow \operatorname{Hom}_{R}\left(\operatorname{Tor}^{S}(R, R), A\right)
$$

defined as follows. Let $\rho$ be an element of $H\left(\operatorname{Hom}_{R}\left(R \otimes_{S} X, A\right)\right)$. Then $\rho$ is represented by an element $u \in \operatorname{Hom}_{R}\left(R \otimes_{S} X, A\right)$ that annihilates $d\left(R \otimes_{S} X\right)$, where $d$ is the boundary map in the complex $R \otimes_{S} X$. Hence, by restriction to the cycles of $R \otimes_{S} X, u$ yields an element of $\operatorname{Hom}_{R}\left(\operatorname{Tor}^{S}(R, R), A\right)$, and it is seen immediately that this element depends only on $\rho$ and not on the particular choice of the representative $u$. Now $h(\rho)$ is defined to be this element of $\operatorname{Hom}_{R}\left(\operatorname{Tor}^{S}(R, R), A\right)$.

Clearly, $h$ is an $R$-module homomorphism of $\operatorname{Ext}_{S}(R, A)$ into $\operatorname{Hom}_{R}\left(\operatorname{Tor}^{S}(R, R), A\right)$. In degree 0 , we have $\operatorname{Tor}_{0}^{S}(R, R)=R \otimes_{S} R=R$, and $\operatorname{Ext}_{S}^{0}(R, A)=\operatorname{Hom}_{S}(R, A)=\operatorname{Hom}_{R}(R, A)$, and this last identification transports $h$ into the identity map. Thus $h$ is an isomorphism in degree 0 . Note that $\operatorname{Tor}_{0}^{S}(R, R)=R$ is projective as an $R$-module, whence the following lemma implies, in particular, that $h$ is an isomorphism also in degree 1.

Lemмa 4.1. Let $\phi: S \rightarrow R$ be an epimorphism of commutative rings with identity elements, and regard $R$-modules as $S$-modules via $\phi$. Let $A$ be an $R$ -

$\left.{ }^{3}\right)$ This is a special case of $[13$, Th. 4 , etc.], which gave the suggestion for our proof of Theorem 3.1. 
module, and let $k$ be a positive integer. Assume that $\operatorname{Tor}_{i}^{S}(R, R)$ is $R$-projective for all $i<k$. Then the map

$$
h_{i}: \operatorname{Ext}_{S}^{i}(R, A) \rightarrow \operatorname{Hom}_{R}\left(\operatorname{Tor}_{i}^{S}(R, R), A\right),
$$

obtained by restriction of the map $h$ defined above, is an isomorphism, for all $i \leqq k$.

Proof. Let $Z_{i}$ denote the kernel of $d$ in $R \otimes_{S} X_{i}$, and put $B_{i}=d\left(R \otimes_{S} X_{i+1}\right)$, $C_{i}=R \otimes_{S} X_{i}$. We have $Z_{0}=C_{0}$. Suppose that we have already shown, for some $i<k$, that $Z_{i}$ is $R$-projective. Since $\operatorname{Tor}_{i}^{S}(R, R)$ is $R$-projective, the exact sequence $0 \rightarrow B_{i} \rightarrow Z_{i} \rightarrow \operatorname{Tor}_{i}^{S}(R, R) \rightarrow 0$ shows that $B_{i}$ is a direct module summand in $Z_{i}$ and hence is $R$-projective. Hence the exact sequence $0 \rightarrow Z_{i+1}$ $\rightarrow C_{i+1} \rightarrow^{d} B_{i} \rightarrow 0$ shows that $Z_{i+1}$ is a direct module summand in $C_{i+1}$ and hence is $R$-projective. Hence, starting at $i=0$, we conclude that $B_{i}$ is a direct $R$-module summand of $C_{i}$ for all $i<k$, and that $Z_{i}$ is a direct $R$-module summand of $C_{i}$ for all $i \leqq k$.

Now let $i \leqq k$, and let $\rho$ be an element of $\operatorname{Ext}_{S}^{i}(R, A)$ such that $h_{i}(\rho)=0$. Let $u$ be a representative of $\rho$ in $\operatorname{Hom}_{R}\left(C_{i}, A\right)$. Then $u$ vanishes on $Z_{i}$, so that it induces an element $v \in \operatorname{Hom}_{R}\left(B_{i-1}, A\right)$ such that $v \circ d=u$. Since $B_{i-1}$ is a direct $R$-module summand of $C_{i-1}$, $v$ can be extended (trivially) to an element $w \in \operatorname{Hom}_{R}\left(C_{i-1}, A\right)$. Now $u=w \circ d$, which means that $\rho=0$. Thus $h_{i}$ is a monomorphism.

Now let $\gamma \in \operatorname{Hom}_{R}\left(\operatorname{Tor}_{i}^{S}(R, R), A\right)$. We may regard $\gamma$ as an element of Hom $_{R}\left(Z_{i}, A\right)$. Since $Z_{i}$ is a direct $R$-module summand of $C_{i}, \gamma$ can be extended to an element of $\operatorname{Hom}_{R}\left(C_{i}, A\right)$, which represents an element $\rho \in \operatorname{Ext}_{S}^{i}(R, A)$ such that $h_{i}(\rho)=\gamma$. Thus $h_{i}$ is an epimorphism. This completes the proof of Lemma 4.1.

In degree 1 , we have $\operatorname{Tor}_{1}^{S}(R, R)=R \otimes_{S} I$, where $I$ is the kernel of $\phi$, and $\operatorname{Ext}_{S}^{1}(R, A)=\operatorname{Hom}_{S}(I, A)=\operatorname{Hom}_{R}\left(R \otimes_{S} I, A\right)$, this identification being precisely the one obtained from the isomorphism $h_{1}$. Alternatively, we may identify $R \otimes_{S} I$ with $I / I^{2}$ (whose $R$-module structure is naturally induced from its $S$-module structure), and thus we may write, compatibly with $h_{1}$, $\operatorname{Tor}_{1}^{S}(R, R)=I / I^{2}$, and $\operatorname{Ext}_{S}^{1}(R, A)=\operatorname{Hom}_{R}\left(I / I^{2}, A\right)$.

We are particularly interested in the following special case. Let $K$ be a commutative ring with identity, and let $R$ be a commutative $K$-algebra with identity. Let $S$ be the $K$-algebra $R \otimes_{K} R$, and let $\phi$ be the epimorphism $S \rightarrow R$ that sends $x \otimes y$ onto $x y$. Let $T_{R}(A)$ denote the $R$-module of all $K$-linear derivations of $R$ into the $R$-module $A$. We recall the well-known fact that $T_{R}(A)$ is naturally isomorphic with $\operatorname{Ext}_{S}^{1}(R, A)$. Indeed, if $\zeta \in T_{R}(A)$ then $\zeta$ yields an element $\zeta^{*} \in \operatorname{Hom}_{S}(I, A)$ such that $\zeta^{*}\left(\sum x \otimes y\right)=\sum x \cdot \zeta(y)$ $=-\sum y \cdot \zeta(x)$ (since $\sum x y=0$ ). Clearly, the map $\zeta \rightarrow \zeta^{*}$ is an $R$-module homomorphism. If $\zeta^{*}=0$, we have, for every $x \in R, \zeta(x)=\zeta^{*}(1 \otimes x-x \otimes 1)=0$. Thus the map $\zeta \rightarrow \zeta^{*}$ is a monomorphism. If $\gamma \in \operatorname{Hom}_{S}(I, A)$, we define a map $\zeta$ of $R$ into $A$ by putting $\zeta(x)=\gamma(1 \otimes x-x \otimes 1)$. One checks easily that 
$\zeta \in T_{R}(A)$ and $\zeta^{*}=\gamma$. Thus the map $\zeta \rightarrow \zeta^{*}$ is an $R$-module isomorphism of $T_{R}(A)$ onto $\operatorname{Ext}_{S}^{1}(R, A)$.

Regard $S$ as an $R$-module such that $x \cdot(y \otimes z)=(x y) \otimes z$, and let $J$ be the $R$-submodule of $S$ that is generated by the elements of the form $1 \otimes(x y)$ $-x \otimes y-y \otimes x$. The factor module $S / J$ is the $R$-module of the formal differentials of $R$. The map $x \rightarrow d x=$ the coset of $1 \otimes x \bmod J$ is the usual derivation of $R$ into the $R$-module of the formal differentials and, in fact, the definition of these amounts simply to enforcing the rule $d(x y)=x d y+y d x$. Let $D_{R}$ denote the $R$-module of these formal differentials. It is easily verified that the map $S \rightarrow I$ that sends $x \otimes y$ onto $x \otimes y-(x y) \otimes 1$ induces in the natural way an isomorphism of the $R$-module $D_{R}$ onto $I / I^{2}=\operatorname{Tor}_{1}^{S}(R, R)$ [3, Exp. 13]. Tracing through our above definitions and identifications, we see immediately that the duality isomorphism $h_{1}: \operatorname{Ext}_{B}^{1}(R, A) \rightarrow \operatorname{Hom}_{R}\left(\operatorname{Tor}_{1}^{S}(R, R), A\right)$ is transported into the map $T_{R}(A) \rightarrow \operatorname{Hom}_{R}\left(D_{R}, A\right)$ attaching to $\zeta \in T_{R}(A)$ the element $\zeta^{\prime}$ of $\operatorname{Hom}_{R}\left(D_{R}, R\right)$ given by $\zeta^{\prime}\left(\sum x d y\right)=\sum x \cdot \zeta(y)$.

Let $U$ be a multiplicatively closed subset of nonzero elements of $R$ containing the identity element. Let $R_{U}$ denote the corresponding ring of quotients. This is still a $K$-algebra, and we write $S_{U}$ for $R_{U} \otimes_{K} R_{U}$. By an argument almost identical with the localization argument of the proof of Theorem 3.1, we see that $R_{U} \otimes_{R} \operatorname{Tor}^{S}(R, R)$ is naturally isomorphic with $\operatorname{Tor}^{S U}\left(R_{U}, R_{U}\right)$. In particular, we have $R_{U} \otimes_{R} D_{R}$ naturally isomorphic with $D_{R_{U}}$.

It is immediate from Theorems 2.1 and 3.1 that, if $K$ is a perfect field and $R$ is a regular affine $K$-algebra, then $\operatorname{Tor}^{S}(R, R)$ is a finitely generated projective $R$-module. We can use the above results to prove the following converse.

THEOREM 4.1( $\left(^{4}\right)$. Let $K$ be a perfect field and let $R$ be an affine $K$-algebra. Put $S=R \otimes_{K} R$ and suppose that $\operatorname{Tor}_{1}^{S}(R, R)$ is $R$-projective. Then $R$ is a regular ring.

Proof. Let $Q$ be the field of quotients of $R$, and let $N$ be a maximal ideal of $R$. Since $S$ is Noetherian, $D_{R}\left(=I / I^{2}\right)$ is finitely generated, and, by assumption, it is $R$-projective. Hence $R_{N} \otimes_{R} D_{R}$ is a finitely generated projective, and hence free $R_{N}$-module. Thus $D_{R_{N}}$ is a finitely generated free $R_{N}$-module. Now $Q \otimes_{R_{N}} D_{R_{N}}$ is isomorphic, as a $Q$-space, with $D_{Q}$. We have $\operatorname{Hom}_{Q}\left(D_{Q}, Q\right)$ isomorphic with $T_{Q}(Q)$, and, since $Q$ is a finitely generated separable extension field of $K, T_{Q}(Q)$ is of dimension $t$ over $Q$, where $t$ is the transcendence degree of $Q$ over $K$. Hence the dimension of $D_{Q}$ over $Q$ is equal to $t$, whence we conclude that $D_{R_{N}}$ is of rank $t$ over $R_{N}$.

Write $L$ for $R_{N}$ and $M$ for $N R_{N}$. Since $D_{L}$ is a free $L$-module of rank $t$, the $L / M$-space $\operatorname{Hom}_{L}\left(D_{L}, L / M\right)$ is of dimension $t$ over $L / M$. We know from the

(4) The essential, local, part of this result is contained in [10, Folgerung, p. 177]. Our proof of the local part is adapted from [3, Exp. 17, Th. 5]. The global theorem has also been obtained by Y. Nakai, On the theory of differentials in commutative rings, J. Math. Soc. Japan 13 (1961), 63-84. 
above that $\operatorname{Hom}_{L}\left(D_{L}, L / M\right)$ is isomorphic with $T_{L}(L / M)$. Hence $T_{L}(L / M)$ is of dimension $t$ over $L / M$. Now a derivation of $L$ in to $L / M$ must annihilate $M^{2}$ and hence induces a derivation of $L / M^{2}$ into $L / M$. Moreover, every derivation of $L / M^{2}$ into $L / M$ can evidently be lifted to give a derivation of $L$ into $L / M$. Hence $T_{L / M^{2}}(L / M)$ is isomorphic, as an $L / M$-space with $T_{L}(L / M)$, and thus is of dimension $t$.

Now $L / M^{2}$ is a finite dimensional algebra over the perfect field $K$ with radical $M / M^{2}$. Hence we can write $L / M^{2}$ as a semidirect sum $L / M^{2}=V$ $+M / M^{2}$, where $V$ is a subalgebra isomorphic with the field $L / M$. Since $K$ is perfect, every $K$-linear derivation of $V$ into $L / M$ must therefore be 0 . Hence it is clear that the restriction of the elements of $T_{L / M^{2}}(L / M)$ to $M / M^{2}$ yields an isomorphism of $T_{L / M^{2}}(L / M)$ onto $\operatorname{Hom}_{L / M}\left(M / M^{2}, L / M\right)$. Hence we conclude that the dimension of $M / M^{2}$ over $L / M$ is equal to $t$. It follows by a standard argument from this that $M$ can be generated by $t$ elements. Since $t$ is the Krull dimension of $L$, this shows that $L$, i.e., $R_{N}$, is a regular local ring. This completes the proof.

5. Explicit multiplication. Let $K$ be a commutative ring with identity, and let $R$ be a commutative $K$-projective $K$-algebra. As before, let $S=R \otimes_{K} R$, and let $\phi$ be the natural epimorphism $S \rightarrow R$. If $A$ and $B$ are $S$-modules we regard them as two sided $R$-modules in the usual way, and we form $A \otimes_{R} B$. This is again a two sided $R$-module, and hence an $S$-module. Let $X$ be an $S$-projective resolution of $R$. Using that $R$ is $K$-projective, we see that $X \otimes_{R} X$ is $S$-projective; essentially, this follows from the fact that $S \otimes_{R} S$ $=R \otimes_{K} R \otimes_{K} R$, with the two sided $R$-module structure in which $a \cdot(u \otimes v \otimes w)$ $=(a u) \otimes v \otimes w$ and $(u \otimes v \otimes w) \cdot a=u \otimes v \otimes(w a)$, so that $S \otimes_{R} S$ is $S$-projective whenever $R$ is $K$-projective. Moreover, $S$ is $R$-projective as a left or right $R$-module, so that $X$ is an $R$-projective resolution of $R$. Hence $H\left(X \otimes_{R} X\right)=\operatorname{Tor}^{R}(R, R)$ and therefore has its components of positive degree equal to 0 , so that $X \otimes_{R} X$ is still an $S$-projective resolution of $R$. For two sided $R$-modules $U$ and $V$, regard $\operatorname{Hom}_{S}(U, V)$ as a two sided $R$-module such that $(r \cdot f)(u)=r \cdot f(u)(=f(r \cdot u))$ and $(f \cdot r)(u)=f(u) \cdot r(=f(u \cdot r))$.

Now the standard $S$-module homomorphism

$$
\psi: \operatorname{Hom}_{S}(X, A) \otimes_{R} \operatorname{Hom}_{S}(X, B) \rightarrow \operatorname{Hom}_{S}\left(X \otimes_{R} X, A \otimes_{R} B\right),
$$

where $\psi(f \otimes g)(u \otimes v)=f(u) \otimes g(v)$, induces an $S$-module homomorphism

$$
\operatorname{Ext}_{S}(R, A) \otimes_{R} \operatorname{Ext}_{S}(R, B) \rightarrow \operatorname{Ext}_{S}\left(R, A \otimes_{R} B\right) .
$$

This is the product $V$, as given in [4, Ex. 2, p. 229], and it is independent of the choice of the resolution $X$. In particular, for $A=B=R$, this defines the structure of an associative and skew-commutative $R$-algebra on $\operatorname{Ext}_{S}(R, R)$.

In order to make the algebra structures on $\operatorname{Tor}^{S}(R, R)$ and $\operatorname{Ext}_{S}(R, R)$ explicit, we use the following well-known resolution $Y$ of $R$ as an $S$-module. We put $Y_{0}=S$ and we let $\phi: S \rightarrow R$ be the augmentation. Generally, let $Y_{n}$ be 
the tensor product, relative to $K$, of $n+2$ copies of $R$. The $S$-module structure of $Y_{n}$ is defined so that

$$
(a \otimes b) \cdot\left(x_{0} \otimes \cdots \otimes x_{n+1}\right)=\left(a x_{0}\right) \otimes x_{1} \otimes \cdots \otimes x_{n} \otimes\left(x_{n+1} b\right) .
$$

The boundary map $d$ on $Y$ is given by

$$
d\left(x_{0} \otimes \cdots \otimes x_{n+1}\right)=\sum_{i=0}^{n}(-1)^{i} x_{0} \otimes \cdots \otimes\left(x_{i} x_{i+1}\right) \otimes \cdots \otimes x_{n+1} .
$$

This complex is not only acyclic but it has actually a right $R$-module homotopy $h$, where $h\left(x_{1} \otimes \cdots \otimes x_{n}\right)=1 \otimes x_{1} \otimes \cdots \otimes x_{n}$. Since $R$ is $K$-projective, it follows as above for $X \otimes_{R} X$ that $Y$ is $S$-projective. Thus $Y$ is an $S$-projective resolution of $R$.

The complex $Y$ can be given the structure of an associative skew-commutative $S$-algebra with respect to which $d$ is an antiderivation, as follows. If $x_{1}, \cdots, x_{p}$ and $y_{1}, \cdots, y_{q}$ are elements of $R$ let $\left[x_{1}, \cdots, x_{p} ; y_{1}, \cdots, y_{q}\right]$ stand for the sum, in the tensor product over $K$ of $p+q$ copies of $R$, of all terms of the form $\pm z_{1} \otimes \cdots \otimes z_{p+q}$, where $z_{i_{k}}=x_{k}$ for some ordered subset $\left(i_{1}, \cdots, i_{p}\right)$ of $(1, \cdots, p+q)$, and $z_{j_{k}}=y_{k}$ for the ordered complement $\left(j_{1}, \cdots, j_{q}\right)$, and where the sign is + or - according to whether the permutation $\left(i_{1}, \cdots, i_{p}, j_{1}, \cdots, j_{q}\right)$ of $(1, \cdots, p+q)$ is even or odd. Then the product in $Y$ is given by the maps $Y_{p} \otimes_{S} Y_{q} \rightarrow Y_{p+q}$ that send

onto

$$
\left(x_{0} \otimes \cdots \otimes x_{p+1}\right) \otimes_{S}\left(y_{0} \otimes \cdots \otimes y_{q+1}\right)
$$

$$
\left(x_{0} y_{0}\right) \otimes\left[x_{1}, \cdots, x_{p} ; y_{1}, \cdots, y_{q}\right] \otimes\left(y_{q+1} x_{p+1}\right) .
$$

It can be verified directly that this is indeed an associative and skew-commutative product and, if $\alpha$ is homogeneous of degree $p$ and $\beta$ arbitrary, one has $d(\alpha \beta)=d(\alpha) \beta+(-1)^{p} \alpha d(\beta)$; cf. [4, pp. 218-219].

This product evidently induces a product in $R \otimes_{S} Y$, and hence in $\operatorname{Tor}^{S}(R, R)$. By the nature of the definition of the product on $\operatorname{Tor}^{S}(R, R)$, as given earlier in the general case, the product induced from that on $Y$ is the standard product $\Pi$ on $\operatorname{Tor}^{S}(R, R)$.

Next we shall define a map of the complex $Y$ into the complex $Y \otimes_{R} Y$ which will serve to make the product on $\operatorname{Ext}_{S}(R, R)$ explicit. We have $\left(Y \otimes_{R} Y\right)_{p}=\sum_{r=0}^{p} Y_{r} \otimes_{R} Y_{p-r}$. As an $S$-module, each $Y_{r} \otimes_{R} Y_{p \rightarrow r}$ may be identified with the tensor product, relative to $K$, of $p+3$ copies of $R$, i.e., with $Y_{p+1}$. With this understanding, we define an $S$-module homomorphism $\gamma_{r}: Y_{p} \rightarrow Y_{r} \otimes_{R} Y_{p-r}$ such that

$$
\gamma_{r}\left(x_{0} \otimes \cdots \otimes x_{p+1}\right)=x_{0} \otimes \cdots \otimes x_{r} \otimes 1 \otimes x_{r+1} \otimes \cdots \otimes x_{p+1} .
$$

Now the desired map $\gamma: Y \rightarrow Y \otimes_{R} Y$ is defined so that, for $u \in Y_{p}$, the component of $\gamma(u)$ in $Y_{r} \otimes_{R} Y_{p-r}$ is $\gamma_{r}(u)$. It is somewhat lengthy, but not diffcult, to verify that $\gamma$ is compatible with the boundary maps on $Y$ and on 
$Y \otimes_{R} Y$. The product $\mathrm{V}$ on $\operatorname{Ext}_{S}(R, R)$ is induced by the product on $\operatorname{Hom}_{S}(Y, R)$ induced by $\gamma$. In particular, with $\alpha \in \operatorname{Hom}_{\mathcal{S}}\left(Y_{p}, R\right)$ and $\beta \in \operatorname{Hom}_{S}\left(Y_{q}, R\right)$, we have( ${ }^{(5)}$

$$
\begin{aligned}
(\alpha \beta)\left(x_{0}\right. & \left.\otimes \cdots \otimes x_{p+q+1}\right) \\
& =\alpha\left(x_{0} \otimes \cdots \otimes x_{p} \otimes 1\right) \beta\left(1 \otimes x_{p+1} \otimes \cdots \otimes x_{p+q+1}\right) \\
& =x_{0} \alpha\left(1 \otimes x_{1} \otimes \cdots \otimes x_{p} \otimes 1\right) \beta\left(1 \otimes x_{p+1} \otimes \cdots \otimes x_{p+q} \otimes 1\right) x_{p+q+1} .
\end{aligned}
$$

Consider a formal differential $\sum x d y \in D_{R}$. It is easily verified that the corresponding element of $\operatorname{Tor}_{1}^{S}(R, R)$ is represented in $R \otimes_{S} Y_{1}$ by the element $\sum x \otimes_{S}(1 \otimes y \otimes 1)$. On the other hand, let $\zeta \in T_{R}(R)=T_{R}$ (say). Then it is easily seen that its image $\zeta^{*} \in \operatorname{Ext}_{S}^{1}(R, R)$ is represented in $\operatorname{Hom}_{S}\left(Y_{1}, R\right)$ by the element $\zeta^{\prime}$, where $\zeta^{\prime}\left(x_{0} \otimes x_{1} \otimes x_{2}\right)=x_{0} \zeta\left(x_{1}\right) x_{2}$.

Now let $\zeta_{1}, \cdots, \zeta_{n}$ be elements of $T_{R}$, and let $\zeta_{1}^{*} \cdots \zeta_{n}^{*}$ denote the product in $\operatorname{Ext}_{s}^{n}(R, R)$ of their canonical images $\zeta_{i}^{*}$ in $\operatorname{Ext}_{S}^{1}(R, R)$. Then $\zeta_{1}^{*} \cdots \zeta_{n}^{*}$ is represented in $\operatorname{Hom}_{S}\left(Y_{n}, R\right)$ by the product $\zeta_{1}^{\prime} \cdots \zeta_{n}^{\prime}$, as induced from the above map $\gamma$. One sees immediately from the formula written above that

$$
\left(\zeta_{1}^{\prime} \cdots \zeta_{n}^{\prime}\right)\left(x_{0} \otimes \cdots \otimes x_{n+1}\right)=x_{0} \zeta_{1}\left(x_{1}\right) \cdots \zeta_{n}\left(x_{n}\right) x_{n+1} .
$$

Now let $\alpha \in \operatorname{Tor}_{p}^{S}(R, R), \quad \beta \in \operatorname{Tor}_{q}^{S}(R, R)$, and let us compute $h\left(\zeta_{1}^{*} \cdots \zeta_{p+q}^{*}\right)(\alpha \beta)$. Choose representatives $a \in R \otimes_{S} Y_{p}$ and $b \in R \otimes_{S} Y_{q}$ of $\alpha$ and $\beta$, respectively. Then $\alpha \beta$ is represented in $R \otimes_{s} Y_{p+q}$ by the product $a b$. We obtain $h\left(\zeta_{1}^{*} \cdots \zeta_{p+q}^{*}\right)(\alpha \beta)$ by applying the element of $\operatorname{Hom}_{R}\left(R \otimes_{S} Y_{p+q}, R\right)$ that corresponds naturally to $\zeta_{1}^{\prime} \cdots \zeta_{p+q}^{\prime}$ to $a b$. Clearly, the result so obtained is the same as the result one would obtain by performing the shuffling involved in forming $a b$ on the sequence $\zeta_{1}^{\prime}, \ldots, \zeta_{p+q}^{\prime}$ rather than on the arguments $x_{i}$ and $y_{j}$ in the product formula for $a b$. Hence we have

$$
h\left(\zeta_{1}^{*} \cdots \zeta_{p+q}^{*}\right)(\alpha \beta)=\sum_{t} \sigma(t) h\left(\zeta_{t(1)}^{*} \cdots \zeta_{t(p)}^{*}\right)(\alpha) h\left(\zeta_{t(p+1)}^{*} \cdots \zeta_{t(p+q)}^{*}\right)(\beta),
$$

where the summation goes over all those permutations $t$ of $(1, \cdots, p+q)$ for which $t(1)<\cdots<t(p)$ and $t(p+1)<\cdots<t(p+q)$, and where $\sigma(t)$ is the signature of $t$.

Let $A^{p}\left(T_{R}\right)$ denote the $R$-module of all alternating $(R, p)$-linear maps $f$ of $p$-tuples of elements of $T_{R}$ into $R$, where "alternating" is to mean that $f\left(\zeta_{t(1)}, \cdots, \zeta_{t(p)}\right)=\sigma(t) f\left(\zeta_{1}, \cdots, \zeta_{p}\right)$, for every permutation $t$ of the set $(1, \cdots, p)$. Then the duality map $h: \operatorname{Ext}_{S}^{p}(R, R) \rightarrow \operatorname{Hom}_{R}\left(\operatorname{Tor}_{p}^{S}(R, R), R\right)$ yields an $R$-module homomorphism $h^{*}$ of $\operatorname{Tor}_{p}^{s}(R, R)$ into $A^{p}\left(T_{R}\right)$, where

$$
h^{*}(\alpha)\left(\zeta_{1}, \cdots, \zeta_{p}\right)=h\left(\zeta_{1}^{*} \cdots \zeta_{p}^{*}\right)(\alpha) .
$$

Put $A\left(T_{R}\right)=\sum_{p} A^{p}\left(T_{R}\right)$. Then $A\left(T_{R}\right)$ is the usual algebra of the differen-

(5) This is the value-wise product of cochains, such as was used in $[11, \mathrm{Th} .6]$. 
tial forms on $R$, where the multiplication is defined by means of the above shuffling of the arguments $\zeta_{i}$ and summing with the appropriate signatures. Our result is therefore the following.

THEOREM 5.1. Let $K$ be a commutative ring with identity, and let $R$ be a $K$-projective commutative $K$-algebra with identity. Let $S=R \otimes_{K} R$. Then the duality map $h$ of $\operatorname{Ext}_{S}(R, R)$ into $\operatorname{Hom}_{R}\left(\operatorname{Tor}^{S}(R, R), R\right)$ and the multiplication in $\operatorname{Ext}_{S}(R, R)$ yield, in the natural fashion, an $R$-algebra homomorphism $h^{*}$ of $\operatorname{Tor}^{S}(R, R)$ into the $R$-algebra $A\left(T_{R}\right)$ of the differential forms on $R$.

In particular, suppose that $R$ is a regular affine $K$-algebra, where $K$ is a perfect field. Then, in virtue of Theorem 2.1, the assumptions of Theorem 3.1 are satisfied, and we conclude that $\operatorname{Tor}^{S}(R, R)$ is finitely generated and projective as an $R$-module, and may be identified with the exterior $R$-algebra constructed over $\operatorname{Tor}_{1}^{\mathcal{S}}(R, R)$. By imbedding $\operatorname{Tor}_{1}^{\mathcal{S}}(R, R)$ as a direct $R$-module summand in a finitely generated free $R$-module, we see that $\operatorname{Hom}_{R}\left(\operatorname{Tor}^{S}(R, R), R\right)$ is isomorphic in the standard fashion (dual of exterior algebra $\approx$ exterior algebra over dual) with the exterior $R$-algebra constructed over $\operatorname{Hom}_{R}\left(\operatorname{Tor}_{1}^{S}(R, R), R\right)$.

Let $E\left(T_{R}\right)$ denote the exterior $R$-algebra constructed over $T_{R}$. The map $\zeta \rightarrow h\left(\zeta^{*}\right)$ is an isomorphism of $T_{R}$ onto $\operatorname{Hom}_{R}\left(\operatorname{Tor}_{1}^{S}(R, R), R\right)$. By what we have just remarked, this extends in the standard fashion to an isomorphism $\rho$ of $E\left(T_{R}\right)$ onto $\operatorname{Hom}_{R}\left(\operatorname{Tor}^{S}(R, R), R\right)$. Let $\zeta_{1}, \cdots, \zeta_{p}$ be elements of $T_{R}$, and let $\zeta_{1} \cdots \zeta_{p}$ stand for their product in $E\left(T_{R}\right)$. Then we see from Theorem 5.1 that $h\left(\zeta_{1}^{*} \cdots \zeta_{p}^{*}\right)=\rho\left(\zeta_{1} \cdots \zeta_{p}\right)$. By Lemma $4.1, h$ is an isomorphism. Hence we conclude that the map $\zeta \rightarrow \zeta^{*}$ extends in the natural fashion to an $R$-algebra isomorphism of $E\left(T_{R}\right)$ onto $\operatorname{Ext}_{S}(R, R)$. Moreover, it is clear that, in the present case, $h^{*}$ is a monomorphism sending $\operatorname{Tor}^{S}(R, R)$ onto the subalgebra of $A\left(T_{R}\right)$ that is generated by the strongly alternating maps, i.e., by the maps that vanish whenever two of the arguments are equal. We may summarize these results as follows.

THEOREM 5.2. Let $R$ be a regular affine $K$-algebra, where $K$ is a perfect field. Then $\operatorname{Tor}^{S}(R, R)$ is naturally isomorphic with the exterior algebra $E\left(D_{R}\right)$ constructed over the $R$-module $D_{R}$ of the formal differentials, and $\operatorname{Ext}_{S}(R, R)$ is naturally isomorphic with the exterior algebra $E\left(T_{R}\right)$ constructed over the $R$-module $T_{R}$ of the $K$-derivations of $R$. These isomorphisms transport the duality map $h: \operatorname{Ext}_{S}(R, R) \rightarrow \operatorname{Hom}_{R}\left(\operatorname{Tor}^{S}(R, R), R\right)$ into the canonical homomorphism $E\left(T_{R}\right) \rightarrow \operatorname{Hom}_{R}\left(E\left(D_{R}\right), R\right)$, which is an isomorphism because $D_{R}$ is finitely generated and projective as an $R$-module. The homomorphism $h^{*}$ of Theorem 5.1 becomes an isomorphism of $E\left(D_{R}\right)$ onto the $R$-algebra of the strongly alternating differential forms.

Now suppose that $K$ is an arbitrary field, and that $F$ is a finitely generated separable extension field of $K$. Then everything we have said above concerning the regular affine $K$-algebra $R$ holds equally for $F$, the only change in the 
proof being that the appeal to Theorem 2.1 is now replaced with an appeal to Theorem 2.2. Thus we have the following result.

THEOREM 5.3. The conclusions of Theorem 5.2 hold also when $K$ is an arbitrary field and $R$ is a finitely generated separable extension field of $K$.

In his thesis (Chicago, 1956), W. Ballard has obtained a part of Theorem 5.3, namely: $\operatorname{Ext}_{S}(R, R) \approx E\left(T_{R}\right)$.

It is of interest to observe, in connection with Theorem 5.1, that the weak definition of "alternating" used in describing $A\left(T_{R}\right)$, rather than the usual stronger requirement on "alternating" maps, which demands that they vanish whenever two of the arguments are equal, is appropriate, in general. This is shown by the following example. Let $K$ be a field of characteristic 2 and let $R=K[a]$, with $a^{2}=u \in K$, but $a \in K$. Consider the element $1 \otimes_{S}(1 \otimes a \otimes a \otimes a)$ $+1 \otimes \otimes_{S}(1 \otimes u \otimes a \otimes 1) \in R \otimes_{S} Y_{2}$. One checks immediately that this element is a cycle and thus represents an element $\alpha \in \operatorname{Tor}_{2}^{\mathcal{S}}(R, R)$. We have $T_{R}=R \zeta$, where $\zeta(a)=1$. Now one verifies immediately that $h^{*}(\alpha)(\zeta, \zeta)=a$. Thus $h^{*}(\alpha)$ is not alternating in the strong sense. Of course, it is clear from Theorem 5.2 that this phenomenon cannot arise when $R$ is a regular affine algebra over a perfect field. Note that in our present example the element $\alpha$ does not belong to the subalgebra of $\operatorname{Tor}^{S}(R, R)$ that is generated by $\operatorname{Tor}_{1}^{S}(R, R)$; indeed, $h^{*}$ must evidently vanish on $\operatorname{Tor}_{1}^{S}(R, R) \operatorname{Tor}_{1}^{S}(R, R)$, in the present case.

6. The algebra of differential operators. Let $K$ be a commutative ring with identity, and let $R$ be a commutative $K$-algebra with identity. Let $T_{R}$ denote the $R$-module of all $K$-derivations of $R$. Clearly, $T_{R}$ has naturally the structure of a Lie algebra over $K$. We make the direct $R$-module sum $R+T_{R}$ into a Lie algebra over $K$, defining the commutators by the formula $\left[r_{1}+\tau_{1}, r_{2}+\tau_{2}\right]=\left(\tau_{1}\left(r_{2}\right)-\tau_{2}\left(r_{1}\right)\right)+\left[\tau_{1}, \tau_{2}\right]$, where $r_{i} \in R, \tau_{i} \in T_{R}$, and $\left[\tau_{1}, \tau_{2}\right]$ is the ordinary commutator $\tau_{1} \tau_{2}-\tau_{2} \tau_{1}$ of the derivations $\tau_{1}$ and $\tau_{2}$.

Let $U$ denote the universal enveloping algebra of the $K$-Lie algebra $R+T_{R}$, defined as the appropriate homomorphic image of the tensor $K$-algebra constructed over the $K$-module $R+T_{R}$. Denote the canonical $K$-module homomorphism of $R+T_{R}$ into $U$ by $z \rightarrow z^{\prime}$. Let $U^{+}$denote the subalgebra of $U$ that is generated by these elements $z^{\prime}$. Let $P$ denote the two sided ideal of $U^{+}$that is generated by the elements of the form $r^{\prime} z^{\prime}-(r \cdot z)^{\prime}$, where $r$ ranges over $R, z$ ranges over $R+T_{R}$, and $r \cdot z$ is the $r$-multiple of $z$ for the $R$-module structure of $R+T_{R}$. We define $V_{R}$ as the factor $K$-algebra $U^{+} / P$.

It is clear from this definition that the unitary $V_{R}$-modules are precisely those Lie algebra modules $M$ for the Lie algebra $R+T_{R}$ on which we have $r \cdot(z \cdot m)=(r \cdot z) \cdot m$, for all $r \in R, z \in R+T_{R}, m \in M$, and on which $1 \cdot m=m$, where 1 is the identity element of $R$. We shall call such modules regular $\left(R+T_{R}\right)$-modules. In particular, it is clear that $R$ is a regular $\left(R+T_{R}\right)$ module in the natural way, and one sees easily that the representation of $R+T_{R}$ on $R$ is faithful. Hence the canonical homomorphism of $R+T_{R}$ into 
$V_{R}$ is actually a monomorphism, and we shall accordingly identify $R+T_{R}$ with its image in $V_{R}$.

Let $A$ and $B$ be any two regular $\left(R+T_{R}\right)$-modules. We define the structure of a regular $\left(R+T_{R}\right)$-module on $\operatorname{Hom}_{R}(A, B)$ such that, with $r \in R$, $\tau \in T_{R}, a \in A, h \in \operatorname{Hom}_{R}(A, B)$,

$$
(\boldsymbol{r} \cdot h)(a)=r \cdot h(a) \text { and }(\tau \cdot h)(a)=\tau \cdot h(a)-h(\tau \cdot a) .
$$

The verification that the above conditions for regularity are satisfied presents no difficulties. Thus, if $A$ and $B$ are any two unitary $V_{R}$-modules, this defines the structure of a unitary $V_{R}$-module on $\operatorname{Hom}_{R}(A, B)$.

LEMMA 6.1. Let $B$ be a unitary $V_{R}$-module, and regard $V_{R}$ as a $V_{R}$-module in the natural fashion (the operators being the left multiplications). Then the $V_{R}$-module $\operatorname{Hom}_{R}\left(V_{R}, B\right)$, with the structure defined above, is isomorphic with the $V_{R}$-module $\operatorname{Hom}_{R}\left(V_{R}, B\right)$ in which the module structure is defined in the usual way from the right multiplications in $V_{R}$, i.e., in which $(u \cdot h)(v)=h(v u)$, for all $h \in \operatorname{Hom}_{R}\left(V_{R}, B\right)$ and all $u, v$ in $V_{R}$.

Proof. For every $h \in \operatorname{Hom}_{R}\left(V_{R}, B\right)$, define $h^{*} \in \operatorname{Hom}_{R}\left(V_{R}, B\right)$ by $h^{*}(u)$ $=(u \cdot h)(1)$, where $u \cdot h$ denotes the transform of $h$ by the element $u$ of $V_{R}$, for the first $V_{R}$-module structure of $\operatorname{Hom}_{R}\left(V_{R}, B\right)$. We claim that, for all $u$, $v$ in $V_{R},\left(u \cdot h^{*}\right)(v)=(v \cdot h)(u)$. This is immediately verified from the definitions when $u \in R$. Now suppose that the result has already been established for some $u$ and all $v$. Let $\tau \in T_{R}$. Then we have

$$
\left(\tau u \cdot h^{*}\right)(v)=\tau \cdot\left(\left(u \cdot h^{*}\right)(v)\right)-\left(u \cdot h^{*}\right)(\tau v)=\tau \cdot((v \cdot h)(u))-(\tau v \cdot h)(u)=(v \cdot h)(\tau u) .
$$

Thus our claim follows inductively for all right $R$-multiples $u$ of monomials in elements of $T_{R}$ and hence generally for all $u \in V_{R}$.

In particular, $\left(u \cdot h^{*}\right)(1)=h(u)$, so that $h^{* *}=h$. Thus our map $h \rightarrow h^{*}$ is an additive (actually $K$-linear) involution of $\operatorname{Hom}_{R}\left(V_{R}, B\right)$. Now we have $(v \cdot h)^{*}(u)=(u v \cdot h)(1)=h^{*}(u v)$. This means that our involution $h \rightarrow h^{*}$ transports the $V_{R}$-module structure defined originally into the $V_{R}$-module structure given by $(v \cdot h)(u)=h(u v)$. This completes the proof of Lemma 6.1.

Let $A$ and $B$ be unitary $V_{R}$-modules. Using the induced $R$-module structures on $A$ and $B$ (written on the right or on the left, as the notation requires), we may form the tensor product $A \otimes_{R} B$. We define the structure of a regular $\left(R+T_{R}\right)$-module, and hence that of a unitary $V_{R}$-module, on $A \otimes_{R} B$ such that, for $r \in R, a \in A, b \in B, \tau \in T_{R}, r \cdot(a \otimes b)=(r \cdot a) \otimes b(=a \otimes(r \cdot b))$ and $\tau \cdot(a \otimes b)=(\tau \cdot a) \otimes b+a \otimes(\tau \cdot b)$. It is verified in a straightforward way that these definitions indeed satisfy the conditions for a regular $\left(R+T_{R}\right)$-module.

Lemma 6.2. Let $B$ be a unitary $V_{R}$-module, and regard $V_{R}$ as a $V_{R}$-module by left multiplication and as a right $R$-module by right multiplication. Then the $V_{R}$-module $B \otimes_{R} V_{R}$, with the module structure defined as above, is isomorphic 
with the $V_{R}$-module $V_{R} \otimes_{R} B$ in which the module structure is defined from that of $V_{R}$ alone, i.e., is such that $u \cdot(v \otimes b)=(u v) \otimes b$.

Proof. There is an evident $V_{R}$-module homomorphism $\psi: V_{R} \otimes_{R} B$ $\rightarrow B \otimes_{R} V_{R}$ such that $\psi(u \otimes b)=u \cdot(b \otimes 1)$. We shall show that $\psi$ is actually an isomorphism by exhibiting an inverse.

For this purpose, we must momentarily return to the definition of $V_{R}$ as the factor algebra $U^{+} / P$. The map $r+\tau \rightarrow r-\tau$ is evidently an anti-automorphism of order 2 of the $K$-Lie algebra $R+T_{R}$ and induces, in the natural way, a $K$-linear involution $u \rightarrow u^{*}$ of $U^{+}$such that, for $r \in R$ and $\tau \in T_{R},\left(r^{\prime}\right)^{*}=r^{\prime}$ and $\left(\tau^{\prime}\right)^{*}=-\tau^{\prime}$, and, for arbitrary elements $u$ and $v$ of $U^{+},(u v)^{*}=v^{*} u^{*}$. Our return to $U^{+}$is necessitated by the fact that this involution does not send the ideal $P$ into itself, so that it does not induce an involution of $V_{R}$. Let $P_{1}$ be the two sided ideal of $U^{+}$that is generated by the elements of the form $r_{1}^{\prime} r_{2}^{\prime}-\left(r_{1} r_{2}\right)^{\prime}$, with $r_{1}$ and $r_{2}$ ranging over $R$. Write $W$ for $U^{+} / P_{1}$ and $P^{\prime}$ for $P / P_{1}$. Then the map $r \rightarrow r^{\prime}$, followed by the canonical epimorphism $U^{+} \rightarrow W$, is a homomorphism of $R$ into $W$ and yields the structure of a two sided $R$ module on $W$ in the natural fashion. Our involution $u \rightarrow u^{*}$ of $U^{+}$sends $P_{1}$ into itself and hence induces an involution of $W$ which we still denote by $w \rightarrow w^{*}$. If $z$ is an element of $R+T_{R}$ we shall write $z^{\prime}$ also to denote the canonical image of $z$ in $W$. By copying the above definitions of the $V_{R}$-modules $B \otimes_{R} V_{R}$ and $V_{R} \otimes_{R} B$ with $W$ in the place of $V_{R}$, we define the $W$-modules $B \otimes_{R} W$ and $W \otimes_{R} B$. There is evidently a $K$-module homomorphism $x \rightarrow x^{*}$ of $B \otimes_{R} W$ into $W \otimes_{R} B$ such that $(b \otimes u)^{*}=u^{*} \otimes b$. Now define the map $\phi: B \otimes_{R} W \rightarrow W \otimes_{R} B$ such that $\phi(b \otimes u)=\left(u^{*} \cdot\left(b \otimes 1^{\prime}\right)\right)^{*}$.

We claim that $\phi$ is a $W$-module homomorphism. Let $r \in R$. Then we have

$$
\begin{aligned}
\phi\left(r^{\prime} \cdot(b \otimes u)\right) & =\phi((r \cdot b) \otimes u)=\left(u^{*} \cdot\left((r \cdot b) \otimes 1^{\prime}\right)\right)^{*} \\
& =\left(u^{*} \cdot\left(b \otimes r^{\prime} 1^{\prime}\right)\right)^{*}=\left(u^{*} \cdot\left(b \otimes 1^{\prime} r^{\prime}\right)\right)^{*} \\
& =r^{\prime} \cdot\left(u^{*} \cdot\left(b \otimes 1^{\prime}\right)\right)^{*}=r^{\prime} \cdot \phi(b \otimes u) .
\end{aligned}
$$

Let $\tau \in T_{R}$. Then we have

$$
\begin{aligned}
\phi\left(\tau^{\prime} \cdot(b \otimes u)\right) & =\phi((\tau \cdot b) \otimes u)+\phi\left(b \otimes\left(\tau^{\prime} u\right)\right) \\
& =\left(u^{*} \cdot\left((\tau \cdot b) \otimes 1^{\prime}\right)\right)^{*}-\left(\left(u^{*} \tau^{\prime}\right) \cdot\left(b \otimes 1^{\prime}\right)\right)^{*}=-\left(u^{*} \cdot\left(b \otimes \tau^{\prime} 1^{\prime}\right)\right)^{*} \\
& =-\left(u^{*} \cdot\left(b \otimes 1^{\prime} \tau^{\prime}\right)\right)^{*} \\
& =\tau^{\prime} \cdot\left(u^{*} \cdot\left(b \otimes 1^{\prime}\right)\right)^{*}=\tau^{\prime} \cdot \phi(b \otimes u) .
\end{aligned}
$$

This suffices to establish our claim.

Now we shall show that $\phi$ maps the canonical image of $B \otimes_{R} P^{\prime}$ in $B \otimes_{R} W$ into the canonical image of $P^{\prime} \otimes_{R} B$ in $W \otimes_{R} B$. We recall that $P$ is the two sided ideal of $U^{+}$that is generated by the elements of the form $r^{\prime} z^{\prime}-(r z)^{\prime}$, where $r \in R$ and $z \in R+T_{R}$. Actually, $P$ coincides with the right ideal that is generated by these elements. In order to see this, it suffices to show that the 
$K$-subspace spanned by the elements of the form $r^{\prime} z^{\prime}-(r z)^{\prime}$ is stable under commutation with the elements of $R+T_{R}$. Now let $z_{1} \in R+T_{R}$. Then we have

$$
\begin{aligned}
{\left[z_{1}^{\prime}, r^{\prime} z^{\prime}-(r z)^{\prime}\right] } & =\left[z_{1}^{\prime}, r^{\prime}\right] z^{\prime}+r^{\prime}\left[z_{1}^{\prime}, z^{\prime}\right]-\left[z_{1}^{\prime},(r z)^{\prime}\right] \\
& =\left[z_{1}, r\right]^{\prime} z^{\prime}+r^{\prime}\left[z_{1}, z\right]^{\prime}-\left[z_{1}, r z\right]^{\prime} \\
& =\left(\left[z_{1}, r\right]^{\prime} z^{\prime}-\left(\left[z_{1}, r\right] z\right)^{\prime}\right)+\left(r^{\prime}\left[z_{1}, z\right]^{\prime}-\left(r\left[z_{1}, z\right]\right)^{\prime}\right),
\end{aligned}
$$

and this is indeed of the required form. Next we observe that $\left(r^{\prime} z^{\prime}-(r z)^{\prime}\right)$ $\cdot(b \otimes u)=b \otimes\left(\left(r^{\prime} z^{\prime}-(r z)^{\prime}\right) u\right)$, as is easily verified directly from the definition of the $W$-module structure on $B \otimes_{R} W$. Since $\phi$ is a $W$-module homomorphism, this shows that $\phi$ maps the element on the right into the canonical image of $P^{\prime} \otimes_{R} B$ in $W \otimes_{R} B$. Hence we may now conclude that $\phi$ maps the image of $B \otimes_{R} P^{\prime}$ in $B \otimes_{R} W$ into the image of $P^{\prime} \otimes_{R} B$ in $W \otimes_{R} B$. Hence $\phi$ induces a map $\gamma$ of $B \otimes_{R} V_{R}$ into $V_{R} \otimes_{R} B$. Since $\phi$ is a $W$-module homomorphism, it is clear that $\gamma$ is a $V_{R}$-module homomorphism. It follows immediately from this that $\gamma \circ \psi$ is the identity map on $V_{R} \otimes_{R} B$.

There remains to prove that $\psi \circ \gamma$ is the identity map on $B \otimes_{R} V_{R}$. It is immediate that $(\psi \circ \gamma)(b \otimes u)=b \otimes u$ whenever $u \in R$. Suppose that we have already shown that this holds for some $u \in V_{R}$ and all $b \in B$. Then we have, with $\tau \in T_{R}$,

$$
\begin{aligned}
(\psi \circ \gamma)(b \otimes(\tau u)) & =(\psi \circ \gamma)(\tau \cdot(b \otimes u)-(\tau \cdot b) \otimes u) \\
& =\tau \cdot(\psi \circ \gamma)(b \otimes u)-(\tau \cdot b) \otimes u \\
& =\tau \cdot(b \otimes u)-(\tau \cdot b) \otimes u=b \otimes(\tau u) .
\end{aligned}
$$

Hence it follows by an evident induction on the "degree" of $u$, written as a polynomial in elements of $T_{R}$, that $\psi \circ \gamma$ is indeed the identity map. This completes the proof of Lemma 6.2.

Now let us assume that $K$ is a field and that $R$ is an affine $K$-algebra. Let $Q$ denote the field of quotients of $R$. Since every $K$-derivation of $R$ extends uniquely to a $K$-derivation of $Q$, we have a natural injection $T_{R} \rightarrow T_{Q}$, and hence a canonical $Q$-linear map $Q \otimes_{R} T_{R} \rightarrow T_{Q}$. Since $R$ is finitely ring-generated over $K$, we see immediately that this map is an epimorphism. Since $Q$ is $R$ flat, the map $Q \otimes_{R} T_{R} \rightarrow Q \otimes_{R} T_{Q}$ induced by the injection $T_{R} \rightarrow T_{Q}$ is a monomorphism. Evidently, the canonical map $Q \otimes_{R} T_{Q} \rightarrow T_{Q}$ is an isomorphism. Thus the canonical map $Q \otimes_{R} T_{R} \rightarrow T_{Q}$ is an isomorphism. We shall identify $Q \otimes_{R} T_{R}$ with $T_{Q}$ whenever convenient.

The injection $R+T_{R} \rightarrow Q+T_{Q}$ is both a Lie algebra homomorphism and an $R$-module homomorphism, and hence extends uniquely to a $K$-algebra homomorphism $V_{R} \rightarrow V_{Q}$. This induces a canonical $Q$-linear epimorphism

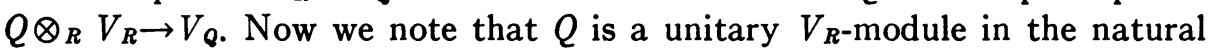
fashion, so that we may equip $Q \otimes_{R} V_{R}$ with the structure of a unitary $V_{R^{-}}$ module, in the manner explained above. The induced $R$-module structure coincides with the $R$-module structure induced by the natural $Q$-module 
structure of $Q \otimes_{R} V_{R}$. We define a product on $Q \otimes_{R} V_{R}$ by means of this $V_{R^{-}}$ module structure and the natural $Q$-module structure, the definition being such that

$$
\left(q_{1} \otimes v_{1}\right)\left(q_{2} \otimes v_{2}\right)=q_{1} \cdot\left(v_{1} \cdot\left(q_{2} \otimes v_{2}\right)\right) .
$$

It is not difficult to verify, by the same kind of induction we used in the proof of Lemma 6.2, that this gives the structure of an associative $K$-algebra on $Q \otimes_{R} V_{R}$. Moreover, it is then clear that our $Q$-linear epimorphism $Q \otimes_{R} V_{R}$ $\rightarrow V_{Q}$ is actually a $K$-algebra epimorphism.

For every non-negative integer $m$, let $V_{R}^{m}$ denote the $R$-submodule of $V_{R}$ consisting of the elements that can be written as sums of products of elements of $R$ and $T_{R}$, where each product has at most $m$ factors from $T_{R}$. If $m$ is a negative integer, let $V_{R}^{m}=(0)$. Let $G\left(V_{R}\right)$ be the graded $R$-algebra $\sum_{m} V_{R}^{m} / V_{R}^{m-1}$ obtained from this filtration of $V_{R} ; G\left(V_{R}\right)$ is indeed an $R$-algebra, and not only a $K$-algebra, because the commutation with an element of $R$ sends each $V_{R}^{m}$ into $V_{R}^{m-1}$. Let $S\left(T_{R}\right)$ denote the symmetric $R$-algebra built over the $R$ module $T_{R}$. We have an evident natural $R$-algebra epimorphism $S\left(T_{R}\right)$ $\rightarrow G\left(V_{R}\right)$. In the same way, we define $G\left(V_{Q}\right), S\left(T_{Q}\right)$, and we have the natural $Q$-algebra epimorphism $S\left(T_{Q}\right) \rightarrow G\left(V_{Q}\right)$.

THEOREM 6.1. Let $R$ be an affine $K$-algebra, where $K$ is an arbitrary field, and let $Q$ be the field of quotients of $R$. Let $F$ be an arbitrary extension field of $K$, regarded as a $K$-algebra. Then the canonical epimorphisms $S\left(T_{F}\right) \rightarrow G\left(V_{F}\right)$ and $Q \otimes_{R} V_{R} \rightarrow V_{Q}$ are isomorphisms.

Proof. The first part of the theorem is analogous to the Poincaré-BirkhoffWitt Theorem for universal enveloping algebras of Lie algebras and can be proved by the method in [4, Lemma 3.5, p. 272]: if $\left(\tau_{i}\right)$ is an ordered $F$-basis for $T_{F}$, it is clear from the definition of $V_{F}$ that every element of $V_{F}$ can be written as an $F$-linear combination of ordered monomials $\tau_{i_{1}} \cdots \tau_{i_{n}}$; $i_{1} \leqq \cdots \leqq i_{n}$. Now one shows inductively that $S\left(T_{F}\right)$ can be equipped with the structure of a regular $\left(F+T_{F}\right)$-module such that the transform of $1 \in S\left(T_{F}\right)$ by $v \in V_{F}$ is precisely the element of $S\left(T_{F}\right)$ that is represented by the above standard expression for $v$. This evidently implies that the representation of the elements of $V_{F}$ in this standard form is unique and that the canonical map $S\left(T_{F}\right) \rightarrow G\left(V_{F}\right)$ is an isomorphism.

In order to prove the second part, let us observe first that the isomorphism $Q \otimes_{R} T_{R} \rightarrow T_{Q}$ extends canonically to a $Q$-algebra isomorphism $Q \otimes_{R} S\left(T_{R}\right)$ $\rightarrow S\left(T_{Q}\right)$, because $Q \otimes_{R} S\left(T_{R}\right)$ may be identified with $S\left(Q \otimes_{R} T_{R}\right)$. The natural homomorphism $V_{R} \rightarrow V_{Q}$ induces an $R$-algebra homomorphism $G\left(V_{R}\right)$ $\rightarrow G\left(V_{Q}\right)$ which, in turn, induces a $Q$-algebra homomorphism $Q \otimes_{R} G\left(V_{R}\right)$ $\rightarrow G\left(V_{Q}\right)$. Thus we have an exact and commutative diagram of homomorphisms 


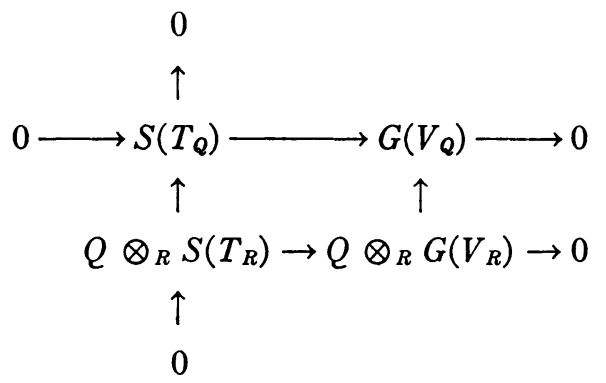

which shows that the homomorphism $Q \otimes_{R} G\left(V_{R}\right) \rightarrow G\left(V_{Q}\right)$ is an isomorphism.

Now let $x$ be an element of $Q \otimes_{R} V_{R}$ such that the image of $x$ in $V_{Q}$ is 0 . If $x \neq 0$, let $m$ be the lowest non-negative integer such that $x$ belongs to the canonical image of $Q \otimes_{R} V_{R}^{m}$ in $Q \otimes_{R} V_{R}$. Then $x$ represents a nonzero element of $Q \otimes_{R}\left(V_{R}^{m} / V_{R}^{m-1}\right) \subset Q \otimes_{R} G\left(V_{R}\right)$. Since the map $Q \otimes_{R} G\left(V_{R}\right) \rightarrow G\left(V_{Q}\right)$ is a monomorphism, this contradicts the assumption that the image of $x$ in $V_{Q}$ is 0 . Hence the epimorphism $Q \otimes_{R} V_{R} \rightarrow V_{Q}$ is indeed an isomorphism, and Theorem 6.1 is proved.

THEOREM 6.2. In the notation of Theorem 6.1, assume that $T_{R}$ is projective as an $R$-module. Then the natural homomorphism $V_{R} \rightarrow V_{Q}$ is a monomorphism, and the canonical epimorphism $S\left(T_{R}\right) \rightarrow G\left(V_{R}\right)$ is an isomorphism.

Proof. Since $T_{R}$ is $R$-projective, so is $S\left(T_{R}\right)$, as is seen by imbedding $T_{R}$ as a direct $R$-module summand in a free $R$-module. Hence the natural homomorphism $S\left(T_{R}\right) \rightarrow Q \otimes_{R} S\left(T_{R}\right)=S\left(T_{Q}\right)$ is a monomorphism. Now we consider the exact and commutative diagram of homomorphisms

$$
\begin{gathered}
0 \rightarrow S\left(T_{Q}\right) \rightarrow G\left(V_{Q}\right) \rightarrow 0 \\
\uparrow \quad \uparrow \\
S\left(T_{R}\right) \rightarrow G\left(V_{R}\right) \rightarrow 0 \\
\uparrow \\
0
\end{gathered}
$$

This shows immediately that the epimorphism $S\left(T_{R}\right) \rightarrow G\left(V_{R}\right)$ is an isomorphism and that the homomorphism $G\left(V_{R}\right) \rightarrow G\left(V_{Q}\right)$ is a monomorphism. The last fact implies as above that the homomorphism $V_{R} \rightarrow V_{Q}$ is a monomorphism, so that Theorem 6.2 is proved.

7. The Ext functor for the algebra of differential operators. If $M$ is any module for a commutative ring, we denote by $E(M)=\sum_{p} E^{p}(M)$ the exterior algebra constructed over $M$. We deal with an affine algebra $R$ over a field $K$, and we let $Q$ denote the field of quotients of $R$. Consider the natural $V_{Q^{-}}$ module $V_{Q} \otimes_{Q} E\left(T_{Q}\right)$, where $u \cdot(v \otimes e)=(u v) \otimes e$. This is graded by the submodules $V_{Q} \otimes_{Q} E^{p}\left(T_{Q}\right)$. Exactly as for the analogous situation of the universal 
enveloping algebra of a Lie algebra [4, Th. 7.1, p. 280], we define a homogeneous $V_{Q}$-endomorphism $d$ of degree -1 on this module such that $d^{2}=0$. This endomorphism is given by the formula

$$
\begin{aligned}
d\left(v \otimes \tau_{0} \cdots \tau_{p}\right)= & \sum_{i=0}^{p}(-1)^{i}\left(v \tau_{i}\right) \otimes \tau_{0} \cdots \hat{\tau}_{i} \cdots \tau_{p} \\
& +\sum_{r<s}(-1)^{r+s} v \otimes\left[\tau_{r}, \tau_{s}\right] \tau_{0} \cdots \hat{\tau}_{r} \cdots \hat{\tau}_{s} \cdots \tau_{p},
\end{aligned}
$$

where the $\tau_{i}$ are arbitrary elements of $T_{Q}$. The definition enforces this formula in the case where the $\tau_{i}$ belong to a given $Q$-basis of $T_{Q}$, and then one verifies easily that the formula holds generally. We augment this complex by the natural $V_{Q}$-module epimorphism $V_{Q} \otimes_{Q} E^{0}\left(T_{Q}\right)=V_{Q} \rightarrow Q$, where the image of $v \in V_{Q}$ in $Q$ is the transform $v \cdot 1$ of $1 \in Q$ by $v$, according to the natural $V_{Q}$ module structure of $Q$. Since, by Theorem $6.1, G\left(V_{Q}\right)$ is isomorphic with $S\left(T_{Q}\right)$, the usual filtration argument [4, pp. 281-282] shows that the augmented complex $V_{Q} \otimes_{Q} E\left(T_{Q}\right)$ is acyclic and is therefore a $V_{Q}$-free resolution of the $V_{Q}$-module $Q$; a sketch of this argument is included in what follows. Note that this conclusion holds more generally for an arbitrary extension field of $K$ in the place of $Q$.

Now let us assume that $T_{R}$ is $R$-projective. Then the same holds for $E\left(T_{R}\right)$. By Theorem 6.2, the natural homomorphism $V_{R} \rightarrow V_{Q}$ is a monomorphism. Hence the induced homomorphism $V_{R} \otimes_{R} E\left(T_{R}\right) \rightarrow V_{Q} \otimes_{R} E\left(T_{R}\right)$ is a monomorphism. Now $V_{Q} \otimes_{R} E\left(T_{R}\right) \equiv V_{Q} \otimes_{Q}\left(Q \otimes_{R} E\left(T_{R}\right)\right)=V_{Q} \otimes_{Q} E\left(Q \otimes_{R} T_{R}\right)$ $=V_{Q} \otimes_{Q} E\left(T_{Q}\right)$. Taking these identifications into due account, we see that our result means that the map $V_{R} \otimes_{R} E\left(T_{R}\right) \rightarrow V_{Q} \otimes_{Q} E\left(T_{Q}\right)$ that is induced by the natural maps $V_{R} \rightarrow V_{Q}$ and $E\left(T_{R}\right) \rightarrow E\left(T_{Q}\right)$ is a monomorphism.

It is seen immediately from the explicit formula for the boundary map $d$ on $V_{Q} \otimes_{Q} E\left(T_{Q}\right)$ that the image of $V_{R} \otimes_{R} E\left(T_{R}\right)$ is stable under $d$. Hence $d$ induces a boundary map (still denoted $d$ ) on $V_{R} \otimes_{R} E\left(T_{R}\right)$, which satisfies the same explicit formula. We have a filtration of this complex by the $R$ subcomplexes $\sum_{q} V_{R}^{p-q} \otimes_{R} E^{q}\left(T_{R}\right)$ (which, since $E\left(T_{R}\right)$ is $R$-projective, may be identified with their canonical images in $V_{R} \otimes_{R} E\left(T_{R}\right)$ ). Now the associated graded complex may evidently be identified with the complex $G\left(V_{R}\right) \otimes_{R} E\left(T_{R}\right)$ and hence, using Theorem 6.2, with the complex $S\left(T_{R}\right) \otimes_{R} E\left(T_{R}\right)$. The boundary operator induced by $d$ (still denoted $d$ ) is given by the formula

$$
d\left(u \otimes \tau_{0} \cdots \tau_{p}\right)=\sum_{i=0}^{p}(-1)^{i}\left(u \tau_{i}\right) \otimes \tau_{0} \cdots \hat{\tau}_{i} \cdots \tau_{p} .
$$

Now let $F$ be a free $R$-module containing $T_{R}$ as a direct $R$-module summand. Then our complex $S\left(T_{R}\right) \otimes_{R} E\left(T_{R}\right)$ is a direct $R$-complex summand of the usual Koszul complex $S(F) \otimes_{R} E(F)$. We shall prove from this that the augmented complex $V_{R} \otimes_{R} E\left(T_{R}\right)$ has an $R$-homotopy. 
As is well known, the augmented complex $S(F) \otimes_{R} E(F)$ has an $R$-homotopy sending $R$ onto $S^{0}(F) \otimes_{R} E^{0}(F)$ and each $S^{p-q}(F) \otimes_{R} E^{q}(F)$ into $S^{p-q-1}(F) \otimes_{R} E^{q+1}(F),\left[8\right.$, p. 259]. Since $S\left(T_{R}\right) \otimes_{R} E\left(T_{R}\right)$ is a direct $R$-complex summand of $S(F) \otimes_{R} E(F)$, the augmented complex $S\left(T_{R}\right) \otimes_{R} E\left(T_{R}\right)$ has an induced $R$-homotopy $h$ sending $R$ onto $S^{0}\left(T_{R}\right) \otimes_{R} E^{0}\left(T_{R}\right)$ and each $S^{p-q}\left(T_{R}\right) \otimes_{R} E^{q}\left(T_{R}\right)$ into $S^{p-q-1}\left(T_{R}\right) \otimes_{R} E^{q+1}\left(T_{R}\right)$. Let $X$ stand for the augmented complex $V_{R} \otimes_{R} E\left(T_{R}\right)$. For $p \geqq 0$, let $X_{p}$ stand for the augmented subcomplex $\sum_{q} V_{R}^{p-q} \otimes_{R} E^{q}\left(T_{R}\right)$, and put $X_{p}=(0)$, for $p<0$. Then the augmented complex $S\left(T_{R}\right) \otimes_{R} E\left(T_{R}\right)$ is the associated graded complex

$$
\sum_{p} X_{p} / X_{p-1}=\sum_{p} G^{p}(X)=G(X) .
$$

Thus we may regard $h$ as an $R$-homotopy of $G(X)$ under which each $G^{p}(X)$ is stable.

Now consider the natural $R$-module epimorphism $X_{p} \rightarrow X_{p} / X_{p-1}=G^{p}(X)$. Since $X_{p} / X_{p-1}$ is isomorphic, as an $R$-module, with $\sum_{q} S^{p-q}\left(T_{R}\right) \otimes_{R} E^{q}\left(T_{R}\right)$, for $p>0$, and since $X_{0}$ is isomorphic, as an $R$-module, with $S^{0}\left(T_{R}\right) \otimes_{R} E^{0}\left(T_{R}\right)$ $+R=R+R$, we know that each $X_{p} / X_{p-1}$ is $R$-projective. Hence we can make a direct $R$-module decomposition $X_{p}=X_{p-1}+Y_{p}$, and $X_{p}$ is the direct $R$ module sum $\sum_{q \leq p} Y_{q}$. If $\alpha_{p}$ denotes the natural $R$-module epimorphism $X_{p} \rightarrow X_{p} / X_{p-1}$ then the restriction of $\alpha_{p}$ to $Y_{p}$ is an isomorphism, and we may define an $R$-module isomorphism $\alpha: X \rightarrow G(X)$ by making $\alpha=\alpha_{p}$ on the component $Y_{p}$. In particular, it follows that $X$ is projective as an $R$-module.

We have $\alpha_{p} d=d \alpha_{p}$. Moreover, $\alpha-\alpha_{p}$ evidently sends $X_{p}$ into $\sum_{q<p} G^{q}(X)$. Hence we have $(\alpha d-d \alpha)\left(X_{p}\right) \subset \sum_{q<p} G^{q}(X)$. Since $G^{p}(X) \subset \alpha\left(X_{p}\right)$, this implies that $\left(d \alpha^{-1}-\alpha^{-1} d\right)\left(G^{p}(X)\right) \subset X_{p-1}$. Put $\gamma=\alpha^{-1} h \alpha$. Then, writing 1 for the identity map, we have

$$
\gamma d+d \gamma-1=\alpha^{-1} h(\alpha d-d \alpha)+\left(d \alpha^{-1}-\alpha^{-1} d\right) h \alpha .
$$

Hence we find that $(\gamma d+d \gamma-1)\left(X_{p}\right) \subset X_{p-1}$. Furthermore, each $X_{p}$ is stable under $\gamma$.

Now we have $-(\gamma d+d \gamma-1)^{2}=\gamma^{\prime} d+d \gamma^{\prime}-1$, where $\gamma^{\prime}=2 \gamma-\gamma d \gamma-d \gamma^{2}$. Hence $\left(\gamma^{\prime} d+d \gamma^{\prime}-1\right)\left(X_{p}\right) \subset X_{p-2}$, and $\left(\gamma^{\prime}-\gamma\right)\left(X_{p}\right) \subset X_{p-1}$. Iteration of this process leads to a sequence of $R$-endomorphisms $\gamma_{k}$ of $X$ such that $\left(\gamma_{k} d+d \gamma_{k}-1\right)\left(X_{p}\right) \subset X_{p-2^{k}}$, and $\left(\gamma_{k+1}-\gamma_{k}\right)\left(X_{p}\right) \subset X_{p-2^{k}}$. Since $X_{q}=(0)$, for $q<0, \gamma_{k+r}-\gamma_{k}$ annihilates $X_{2}{ }^{k}-1$, for all $r \geqq 0$. Hence there is an $R$-endomorphism $\zeta$ on $X$ such that $\zeta$ coincides with $\gamma_{k}$ on $X_{2}{ }^{k}-1$, for each $k$, and we have $\zeta d+d \zeta=1$, i.e., $\zeta$ is an $R$-homotopy of $X$. Thus we have the following result.

Theorem 7.1. Let $R$ be an affine algebra over a field $K$, and suppose that $T_{R}$ is $R$-projective. Then the complex $V_{R} \otimes_{R} E\left(T_{R}\right)$, as defined above, is a $V_{R}$-projective resolution of the $V_{R}$-module $R$ and has an $R$-homotopy. If $F$ is an arbitrary extension field of $K$, regarded as a $K$-algebra, then $V_{F} \otimes_{F} E\left(T_{F}\right)$ is a $V_{F}$-free resolution of the $V_{F-\text { module }} F$. 
Note. It is clear from our proof that Theorem 7.1 holds more generally for any integral domain $R$ over $K$ such that $T_{R}$ is $R$-projective; one must merely replace $T_{Q}$ with the canonical image of $Q \otimes_{R} T_{R}$ in $T_{Q}$. As was pointed out to us by A. Shapiro, further generalizations, covering cases where $R$ is not an integral domain (e.g. the algebra of all differentiable functions on a differentiable manifold), are easily obtainable by simultaneous use of a suitably large family of localizations of $R$. Naturally, these generalizations extend to Corollary 7.1 below.

Let $P=R$, or $P=F$, as in Theorem 7.1, and let $A$ be a unitary $V_{P}$-module. By Theorem 7.1, we have $\operatorname{Ext}_{V_{P}}(P, A)=H\left(\operatorname{Hom}_{V_{P}}\left(V_{P} \otimes_{P} E\left(T_{P}\right), A\right)\right)$. We may identify $\operatorname{Hom}_{V_{P}}\left(V_{P} \otimes_{P} E\left(T_{P}\right), A\right)$ with $\operatorname{Hom}_{P}\left(E\left(T_{P}\right), A\right)$. Let $\delta$ be the coboundary map in $\operatorname{Hom}_{P}\left(E\left(T_{P}\right), A\right)$ that is induced by the boundary map $d$ on $V_{P} \otimes_{P} E\left(T_{P}\right)$. Then, writing the elements of $\operatorname{Hom}_{P}\left(E\left(T_{P}\right), A\right)$ as strongly alternating maps with arguments in $T_{P}$ and values in $A$, we have

$$
\begin{aligned}
(\delta f)\left(\tau_{0}, \cdots, \tau_{p}\right)= & \sum_{i=0}^{p}(-1)^{i} \tau_{i}\left(f\left(\tau_{0}, \cdots, \hat{\tau}_{i}, \cdots, \tau_{p}\right)\right) \\
& +\sum_{r<s}(-1)^{r+s} f\left(\left[\tau_{r}, \tau_{s}\right], \tau_{0}, \cdots, \hat{\tau}_{r}, \cdots, \hat{\tau}_{s}, \cdots, \tau_{p}\right),
\end{aligned}
$$

if $f \in \operatorname{Hom}_{P}\left(E^{p}\left(T_{P}\right), A\right)$. Thus we see that $\operatorname{Ext}_{V_{P}}(P, A)$ is naturally isomorphic with the usual cohomology space based on the strongly alternating $A$-valued differential forms. We state this formally, for reference.

Corollary 7.1. Let $P=R$, or $P=F$, as in Theorem 7.1. Then the cohomology $K$-space based on the strongly alternating differential forms with values in a unitary $V_{P}$-module $A$ may be identified with $\operatorname{Ext}_{V_{P}}(P, A)$.

If we assume that $P$ is either a regular affine $K$-algebra, where $K$ is a perfect field, or that $K$ is an arbitrary field and $P$ is a finitely generated separable extension field of $K$, we may appeal to Theorem 5.2, or to Theorem 5.3 , respectively, to see that the complex of the strongly alternating $P$-valued differential forms may be identified with the complex $E\left(D_{P}\right)$ of the formal differentials, whose differential operator is the canonical extension of the map $x \rightarrow d x$ of $P$ into $D_{P}$. Hence, in this case, the cohomology $K$-space based on the formal differentials of the K-algebra $P$ may be identified, by Corollary 7.1, with $\operatorname{Ext}_{V_{P}}(P, P)\left({ }^{6}\right)$.

\section{The homological dimension of the algebra of differential operators.}

THEOREM 8.1. Let $F$ be a finitely generated extension field of an arbitrary field $K$. Then the global homological dimension $d\left(V_{F}\right)$ of $V_{F}$ is equal to the

( ${ }^{6}$ ) It may be of interest to point out that, in a rather different situation, namely when $P$ is a finitely generated purely inseparable extension field of exponent 1 of $K$, the cohomology $K$-algebra of the strongly alternating $P$-valued differential forms has been determined explicitly by $\mathrm{P}$. Cartier in [5]. 
dimension over $F$ of the space $T_{F}$ of all $K$-derivations of $F$, and is equal to the projective dimension $d_{V_{F}}(F)$ of the $V_{F-m o d u l e ~} F$.

Proof. Let $M$ be any unitary $V_{F}$-module. Then the resolution $V_{F} \otimes_{F} E\left(T_{F}\right)$ of $F$, as in Theorem 7.1, dualizes into an exact sequence of $V_{F}$-modules and $V_{F}$-homomorphisms

$$
\begin{aligned}
(0) \rightarrow M=\operatorname{Hom}_{F}(F, M) & \rightarrow \operatorname{Hom}_{F}\left(V_{F} \otimes_{F} E^{0}\left(T_{F}\right), M\right) \\
& \rightarrow \operatorname{Hom}_{F}\left(V_{F} \otimes_{F} E^{1}\left(T_{F}\right), M\right) \rightarrow \cdots .
\end{aligned}
$$

As a $V_{F}$-module, each $\operatorname{Hom}_{F}\left(V_{F} \otimes_{F} E^{p}\left(T_{F}\right), M\right)$ is isomorphic with a direct sum of a finite number of copies of $\operatorname{Hom}_{F}\left(V_{F}, M\right)$, with the module structure defined just above the statement of Lemma 6.1. However, by Lemma 6.1, this $V_{F}$-module is isomorphic with the $V_{F}$-module $\operatorname{Hom}_{F}\left(V_{F}, M\right)$ with the module structure given by $(u \cdot h)(v)=h(v u)$. As is well known, and easy to show directly (using that $F$ is a field), this module is $V_{F}$-injective. Hence we conclude that the above exact sequence is a $V_{F}$-injective resolution of $M$. Since $E^{p}\left(T_{F}\right)=(0)$ when $p$ exceeds the $F$-dimension of $T_{F}$, it follows that $d\left(V_{F}\right)$ does not exceed the $F$-dimension of $T_{F}$.

Now let $n$ denote the $F$-dimension of $T_{F}$ and let $A$ be any unitary $V_{F^{-}}$ module. There is a basis $\zeta_{1}, \cdots, \zeta_{n}$ for $T_{F}$ over $F$ and elements $x_{1}, \cdots, x_{n}$ in $F$ such that $\zeta_{i}\left(x_{j}\right)=\delta_{i j}\left[7\right.$, Lemma 2.1]. In particular, we have $\left[\zeta_{i}, \zeta_{j}\right]=0$, for all $i$ and $j$. If we use the resolution $V_{F} \otimes_{F} E\left(T_{F}\right)$ of $F$, the $K$-space $\operatorname{Ext}_{V_{F}}^{n}(F, A)$ appears as the factor space

$$
\operatorname{Hom}_{F}\left(E^{n}\left(T_{F}\right), A\right) / \delta\left(\operatorname{Hom}_{F}\left(E^{n-1}\left(T_{F}\right), A\right) .\right.
$$

Using the explicit formula for $\delta$, as given below the statement of Theorem 7.1 , and taking account of the fact that $\left[\zeta_{i}, \zeta_{j}\right]=0$, we see that $\delta\left(\operatorname{Hom}_{F}\left(E^{n-1}\left(T_{F}\right), A\right) \approx T_{F} \cdot A\right.$. On the other hand, $\operatorname{Hom}_{F}\left(E^{n}\left(T_{F}\right), A\right) \approx A$. Hence we see that $\operatorname{Ext}_{V_{F}}^{n}(F, A)$ is isomorphic with $A / T_{F} \cdot A$. Taking $A=V_{F}$, we see from this that $\operatorname{Ext}_{V_{F}}^{n}\left(F, V_{F}\right) \approx F \neq(0)$. Hence we have indeed $d\left(V_{F}\right)$ $=d_{V_{F}}(F)=n$.

Now let $R$ be an affine $K$-algebra such that $T_{R}$ is $R$-projective. In this case, it appears that the relative global homological dimension $d\left(V_{R}, R\right)$ is more easily accessible than $d\left(V_{R}\right)$. We refer to [8] for the requisite notions of relative homological algebra, but we recall that the relative homological notions for $\left(V_{R}, R\right)$ are obtained from the corresponding notions for $V_{R}$ simply by replacing "exact sequence of $V_{R}$-homomorphisms" with " $R$-split sequence of $V_{R}$-homomorphisms" throughout. The ordinary projective dimension of a $V_{R}$-module $M$ will be denoted by $d_{V_{R}}(M)$, and the relative projective dimension of $M$ will be denoted by $d_{\left(V_{R}, R\right)}(M)$. We shall prove the following result.

THEOREM 8.2. Let $K$ be a field and let $R$ be an affine $K$-algebra such that $T_{R}$ is $R$-projective. Let $Q$ be the field of quotients of $R$, and let $n$ be the $Q$-dimension of $T_{Q}$. Then, for every unitary $V_{R}$-module $A$, the canonical map (induced 
from maps of the appropriate resolutions) of $\operatorname{Ext}_{\left(V_{R}, R\right)}(R, A)$ into $\operatorname{Ext}_{V_{R}}(R, A)$ is an isomorphism. We have $d_{V_{R}}(R)=d_{\left(V_{R}, R\right)}(R)=d\left(V_{R}, R\right)=n$. If $R$ is regular then $d\left(V_{R}\right) \leqq n+t$, where $t$ is the transcendence degree of $Q$ over $K$.

Proof. By Theorem 7.1, $V_{R} \otimes_{R} E\left(T_{R}\right)$ is both a $V_{R}$-projective resolution of $R$ and a $\left(V_{R}, R\right)$-projective resolution with $R$-homotopy. Hence both $\operatorname{Ext}_{V_{R}}(R, A)$ and $\operatorname{Ext}_{\left(V_{R}, R\right)}(R, A)$ can be computed as

$$
H\left(\operatorname{Hom}_{V_{R}}\left(V_{R} \otimes_{R} E\left(T_{R}\right), A\right)\right),
$$

which establishes the first assertion of our theorem.

We know that $Q \otimes_{R} E\left(T_{R}\right)$ is isomorphic with $E\left(T_{Q}\right)$. Since $E\left(T_{R}\right)$ is $R$-projective, the natural map $E\left(T_{R}\right) \rightarrow Q \otimes_{R} E\left(T_{R}\right)$ is a monomorphism. Hence we have $E^{p}\left(T_{R}\right)=(0)$ for $p>n$. Hence it is clear from the resolution $V_{R} \otimes_{R} E\left(T_{R}\right)$ of $R$ that $d_{V_{R}}(R)=d_{\left(V_{R}, R\right)}(R) \leqq n$.

Now let us consider the same sequence we used at the beginning of our proof of Theorem 8.1:

$$
\begin{aligned}
(0) \rightarrow M=\operatorname{Hom}_{R}(R, M) & \rightarrow \operatorname{Hom}_{R}\left(V_{R} \otimes_{R} E^{0}\left(T_{R}\right), M\right) \\
& \rightarrow \operatorname{Hom}_{R}\left(V_{R} \otimes_{R} E^{1}\left(T_{R}\right), M\right) \rightarrow \cdots .
\end{aligned}
$$

By Theorem 7.1, the resolution $V_{R} \otimes_{R} E\left(T_{R}\right)$ of $R$ has an $R$-homotopy, which evidently induces an $R$-homotopy of the above dual sequence. On the other hand, we see from Lemma 6.1, as in the proof of Theorem 8.1, that each $\operatorname{Hom}_{R}\left(V_{R} \otimes_{R} E^{p}\left(T_{R}\right), M\right)$ is isomorphic with a direct $V_{R}$-module summand of a direct sum of $V_{R}$-modules $\operatorname{Hom}_{R}\left(V_{R}, M\right)$ with the module structure given by $(v \cdot h)(u)=h(u v)$. By [8, Lemma 1$]$, this last $V_{R}$-module is $\left(V_{R}, R\right)$-injective. Hence the above sequence is a $\left(V_{R}, R\right)$-injective resolution with $R$ homotopy of $M$. Hence it is clear that $d\left(V_{R}, R\right) \leqq n$.

Now we claim that, for every $V_{Q}$-module $M, \operatorname{Ext}_{V_{R}}(R, M)$ is isomorphic with $\operatorname{Ext}_{V_{Q}}(Q, M)$. By Theorem 6.1, $V_{Q}$ is isomorphic with $Q \otimes_{R} V_{R}$. It is clear from the definition of the algebra structure of $Q \otimes_{R} V_{R}$ that this isomorphism transports the right $V_{R}$-module structure of $Q \otimes_{R} V_{R}$ into the right $V_{R}$-module structure of $V_{Q}$ obtained from the natural map of $V_{R}$ into $V_{Q}$. Since $Q$ is $R$-flat, this implies that, as a right $V_{R}$-module, $V_{Q}$ is $V_{R}$-flat. Hence, if $X$ is any $V_{R}$-projective resolution of $R, V_{Q} \otimes_{V_{R}} X$ is a $V_{Q}$-projective resolution of $V_{Q} \otimes_{V_{R}} R$. Using the natural $V_{Q}$-module structure of $Q$, we obtain a $V_{Q}$-module epimorphism $V_{Q} \otimes_{V_{R}} R \rightarrow Q$ sending $v \otimes r$ onto $v \cdot r \in Q$. It is easy to verify that this is actually an isomorphism, so that we may identify $V_{Q} \otimes_{V_{R}} R$ with $Q$, as a $V_{Q}$-module. Thus $V_{Q} \otimes_{V_{R}} X$ is a $V_{Q}$-projective resolution of $Q$. Since $\operatorname{Hom}_{V_{Q}}\left(V_{Q} \otimes_{V_{R}} X, M\right)$ may be identified with $\operatorname{Hom}_{V_{R}}(X, M)$, this establishes our claim. Now it is clear from Theorem 8.1 (applied to $Q$ ) that $d_{V_{R}}(R) \geqq n$. We have shown that $n \leqq d_{V_{R}}(R)=d_{\left(V_{R}, R\right)}(R) \leqq d\left(V_{R}, R\right) \leqq n$, so that all but the last statement of Theorem 8.2 is proved.

As a by-product of our proof of the existence of an $R$-homotopy in 
$V_{R} \otimes_{R} E\left(T_{R}\right)$, we had obtained the result that this complex is $R$-projective. In particular, $V_{k}$ is $R$-projective as a left $R$-module; in fact, as an $R$-module, $V_{R}$ is isomorphic with $G\left(V_{R}\right) \approx S\left(T_{R}\right)$. Similarly, $V_{R}$ is $R$-projective as a right $R$-module. Hence we may apply $[9$, Th. 1] to conclude that, for every unitary $V_{R}$-module $N$,

$$
d_{V_{R}}(N) \leqq d_{\left(V_{R}, R\right)}(N)+d_{R}(N) .
$$

If $R$ is a regular affine $K$-algebra, we have $d\left(R_{M}\right)=t$, for every maximal ideal $M$ of $R$, by $[2$, Ths. $1.9,1.10]$. Since $d(R)=\max _{M}\left(d\left(R_{M}\right)\right)[4$, Ex. 11, p. 142; 1 , Th. 1], we have therefore $d(R)=t$. Hence the above results give $d\left(V_{R}\right)$ $\leqq d\left(V_{R}, R\right)+d(R)=n+t$. This completes the proof of Theorem 8.2.

9. The product for $\operatorname{Ext}_{V_{P}}(P, *)$. Let $K$ be a field, and let $P$ be a $K$-algebra which is either an affine $K$-algebra with $T_{P} P$-projective or an arbitrary extension field of $K$. If $A$ and $B$ are unitary $V_{P}$-modules there is a product

$$
\operatorname{Ext}_{V_{P}}(P, A) \otimes_{K} \operatorname{Ext}_{V_{P}}(P, B) \rightarrow \operatorname{Ext}_{V_{P}}\left(P, A \otimes_{P} B\right)
$$

which is defined as follows. Let $X$ be any $V_{P}$-projective resolution of $P$. Noting that $V_{P}$ is $P$-projective, so that $X$ is $P$-projective, and appealing to Lemma 6.2, we see that the $V_{P}$-module $X \otimes_{P} X$ is still $V_{P}$-projective. Moreover, since $X$ is also a $P$-projective resolution of $P$, we have $H\left(X \otimes_{P} X\right)$ $=\operatorname{Tor}^{P}(P, P)$, whence $H\left(X \otimes_{P} X\right)$ has its components of positive degree equal to (0). Hence $X \otimes_{P} X$ is still a $V_{P}$-projective resolution of $P \otimes_{P} P=P$. Hence the natural $K$-space homomorphism

$$
\phi: \operatorname{Hom}_{V_{P}}(X, A) \otimes_{K} \operatorname{Hom}_{V_{P}}(X, B) \rightarrow \operatorname{Hom}_{V_{P}}\left(X \otimes_{P} X, A \otimes_{P} B\right),
$$

where $\phi(f \otimes g)(u \otimes v)=f(u) \otimes g(v)$, induces a product for $\operatorname{Ext}_{V_{P}}\left(P,{ }^{*}\right)$, as indicated above. It is seen as usual that this product is associative and skewcommutative. In order to make this product explicit, we require a map of the complex $X$ into the complex $X \otimes_{P} X$, when $X=V_{P} \otimes_{P} E\left(T_{P}\right)$. By imbedding $T_{P}$ as a direct $P$-module summand in a free $P$-module, we see easily that there is a $P$-module homomorphism $E^{n}\left(T_{P}\right) \rightarrow E^{p}\left(T_{P}\right) \otimes_{P} E^{n-p}\left(T_{P}\right)$ sending each product $\zeta_{1} \cdots \zeta_{n}$ of elements of $T_{P}$ onto

$$
\sum_{t} \sigma(t) \zeta_{t(1)} \cdots \zeta_{t(p)} \otimes \zeta_{t(p+1)} \cdots \zeta_{t(n)},
$$

where the summation goes over all permutations $t$ of $(1, \cdots, n)$ for which $t(1)<\cdots<t(p)$ and $t(p+1)<\cdots<t(n)$, and where $\sigma(t)$ is the signature of t. Hence there is a map $X \rightarrow X \otimes_{P} X$ sending $v \otimes \zeta_{1} \cdots \zeta_{n} \in V_{P} \otimes_{P} E^{n}\left(T_{P}\right)$ onto

$$
v \cdot\left(\sum_{p=0}^{n}\left(\sum_{t} \sigma(t)\left(1 \otimes \zeta_{t(1)} \cdots \zeta_{t(p)}\right) \otimes\left(1 \otimes \zeta_{t(p+1)} \cdots \zeta_{t(n)}\right)\right)\right) .
$$

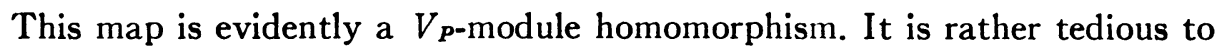
verify that it commutes with the boundary maps on $X$ and $X \otimes_{P} X$, but no 


\section{G. HOCHSCHILD, BERTRAM KOSTANT AND ALEX ROSENBERG}

difficulties other than those of notation are encountered in carrying out this verification by induction on the degree in $X$. On the other hand, it is clear from the definition of this map that our product for $\operatorname{Ext}_{V_{P}}(P, *)$ is the same product as that obtained from the usual shuffle product of alternating differential forms, via the identification of Corollary 7.1.

\section{REFERENCES}

1. M. Auslander, On the dimension of modules and algebras. III, Nagoya Math. J. 9 (1955), 67-77.

2. M. Auslander and D. Buchsbaum, Homological dimension in local rings, Trans. Amer. Math. Soc. 85 (1957), 390-405.

3. H. Cartan et C. Chevalley, Séminaire 1955/56, Ecole Norm. Sup. Paris, 1956.

4. H. Cartan and S. Eilenberg, Homological algebra, Princeton Univ. Press, Princeton, N. J., 1956.

5. P. Cartier, Questions de rationalité des diviseurs en Géométrie algébrique, Bull. Soc. Math. France 86 (1958), 177-251.

6. C. Chevalley, Introduction to the theory of algebraic functions of one variable, Math. Surveys, Amer. Math. Soc., Providence, R. I., 1951. 460.

7. G. Hochschild, Double vector spaces over division rings, Amer. J. Math. 71 (1949), 443-

8. - Relative homological algebra, Trans. Amer. Math. Soc. 82 (1956), 246-269.

9. - Note on relative homological dimension, Nagoya Math. J. 13 (1958), 89-94.

10. E. Kunz, Die Primidealtheiler der Differenten in allgemeinen Ringen, J. Reine Angew. Math. 204 (1960), 165-182.

11. A. Rosenberg and D. Zelinsky, Cohomology of infinite algebras, Trans. Amer. Math. Soc. 82 (1956), 85-98.

12. J.-P. Serre, Sur la dimension des anneaux et des modules noethériens, Proc. Int. Symp. on Algebraic Number Theory, Tokyo-Nikko, 1955, pp. 175-189. 14-27.

13. J. T. Tate, Homology of Noetherian rings and local rings, Illinois J. Math. 1 (1957),

14. O. Zariski and P. Samuel, Commutative algebra, Vol. II, Van Nostrand, Princeton, 1960.

UNIVERSITY OF CALIFORNIA,

Berkeley, California

NORTHWESTERN UNIVERSITY, Evanston, Illinois 